
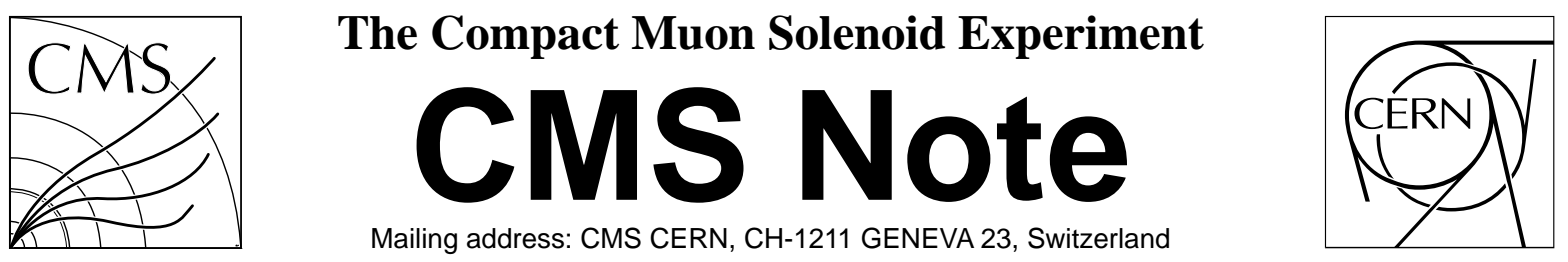

3 Feb. 2006

\title{
Measurement of Missing Transverse Energy With the CMS Detector at the LHC
}

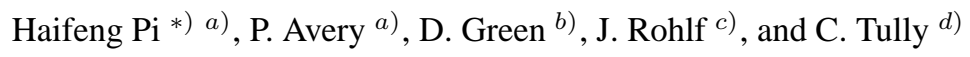 \\ a) University of Florida, Gainesville, FL 32601, USA \\ b) Fermi National Accelerator Laboratory, Batavia, IL 60510, USA \\ c) Boston University, Boston, MA 02215, USA \\ d) Princeton University, Princeton, NJ 08544, USA
}

\begin{abstract}
The performance of the Compact Muon Solenoid detector for measuring missing transverse energy is evaluated using fully simulated pp collisions at a center-of-mass energy of $14 \mathrm{TeV}$ at the Large Hadron Collider. For minimum bias events without pileup, a resolution of $6.1 \mathrm{GeV}$ is computed, corresponding to a stochastic contribution of $0.63 \sqrt{\Sigma E_{\mathrm{T}}} \mathrm{GeV}^{1 / 2}$, where $\Sigma E_{\mathrm{T}}$ is the summed transverse energy in all calorimeter towers. When the contribution of pileup is included, the resolution degrades according to the overall deposited $\Sigma E_{\mathrm{T}}$ with the same stochastic coefficient. For QCD dijet events with event pileup corresponding to a luminosity $\mathcal{L}=2 \times 10^{33} \mathrm{~cm}^{-2} \mathrm{~s}^{-1}$, we compute $\sigma=\left[(3.8 \mathrm{GeV})^{2}+\right.$ $\left.\left(0.97 \mathrm{GeV}^{1 / 2} \sqrt{\Sigma E_{\mathrm{T}}}\right)^{2}+\left(0.012 \Sigma E_{\mathrm{T}}\right)^{2}\right]^{1 / 2}$ resulting in a resolution of $45 \mathrm{GeV}$ for jet events with reconstructed transverse momentum of $800 \mathrm{GeV} / c$. Monte Carlo samples of $t \bar{t}$ and $\mathrm{W}+$ jet events with high-momentum $\left(p_{T}>20 \mathrm{GeV} / c\right)$ lepton decays leading to true missing transverse energy were used to determine the azimuthal angle resolution to be 0.1 radians ( 0.2 radians) for a reconstructed missing transverse energy of $200 \mathrm{GeV}(100 \mathrm{GeV})$.
\end{abstract}

\footnotetext{
*) Work done in partial requirement to fulfill the $\mathrm{PhD}$ degree.
} 


\section{Introduction}

The understanding of detector response to standard model physics from quantum chromodynamics (QCD) is a prerequisite to the search for new phenomena at the Large Hadron Collider (LHC) [1]. Beginning with UA1 [2], all major detectors at hadron colliders have been designed to cover as much solid angle as practically possible with calorimetry. This was a major consideration in the design of calorimetry [3] for the Compact Muon Solenoid (CMS) [4]. The primary motivation is to provide as complete of a picture as possible of the event, including the presence of one or more energetic neutrinos or other weakly-interacting stable particles though apparent missing energy. Energetic particles produced in the direction of the beam pipe make it impossible to directly measure missing energy longitudinal to the beam direction, however, the transverse energy balance can be measured with an accuracy good enough to help establish a physics signature involving one or more non-interacting particles. The $\mathrm{W}$ boson was discovered and its mass determined to $3 \%$ with just 6 events due to the ability of UA1 to infer the presence of 40-GeV neutrinos with a resolution of a few $\mathrm{GeV}$ [5]. Since the time of the $\mathrm{W}$ discovery, measurement of missing transverse energy has been a major tool in the search for new phenomena at hadron colliders [6]-[9].

Measurement of the missing transverse energy vector $\left(\mathbf{E}_{\mathrm{T}}^{\mathrm{miss}}\right)$ in events at the LHC will be complicated by the presence of pileup collisions. In CMS, measurement of $\mathbf{E}_{\mathrm{T}}^{\text {miss }}$ will be further degraded by the difference between photon and pion response in the calorimeters and by the bending of tracks by the 4-T magnetic field. On the other hand, the excellent cell segmentation, hermeticity, and good forward coverage of CMS will help the measurement of $\mathbf{E}_{\mathrm{T}}^{\text {miss }}$ [3].

\section{Calculation of Missing Transverse Energy in CMS}

Readout cells in the CMS hadron calorimeter (HCAL) are arranged in a tower pattern in $\eta, \varphi$ space, projective to the nominal interaction point. The HCAL cells have a segmentation of $0.087 \times 0.087$ in the central region $(|\eta|<1.74)$ and approximately $0.17 \times 0.17$ for $1.74<|\eta|<5$. Since the granularity of the electromagnetic crystal calorimeter $(\mathrm{ECAL})[10]$ is much finer $(0.017 \times 0.017)$ than HCAL, calorimeter towers (ECAL plus HCAL) are formed by addition of signals in $\eta, \varphi$ bins corresponding to the HCAL geometry. In forming the towers, individual calorimeter cells must pass energy threshold cuts of $500 \mathrm{MeV}$ for HCAL cells, $90 \mathrm{MeV}$ for ECAL barrel $(|\eta|<1.48)$ and 450 $\mathrm{MeV}$ for ECAL endcap crystals $(1.48<|\eta|<3.00)$. The thresholds correspond to roughly 2-3 standard deviations above the expected root-mean-square electronics noise. In total there are 4176 such towers, which when unfolded, may be represented in a familiar "lego" plot (Fig. 1) [11].

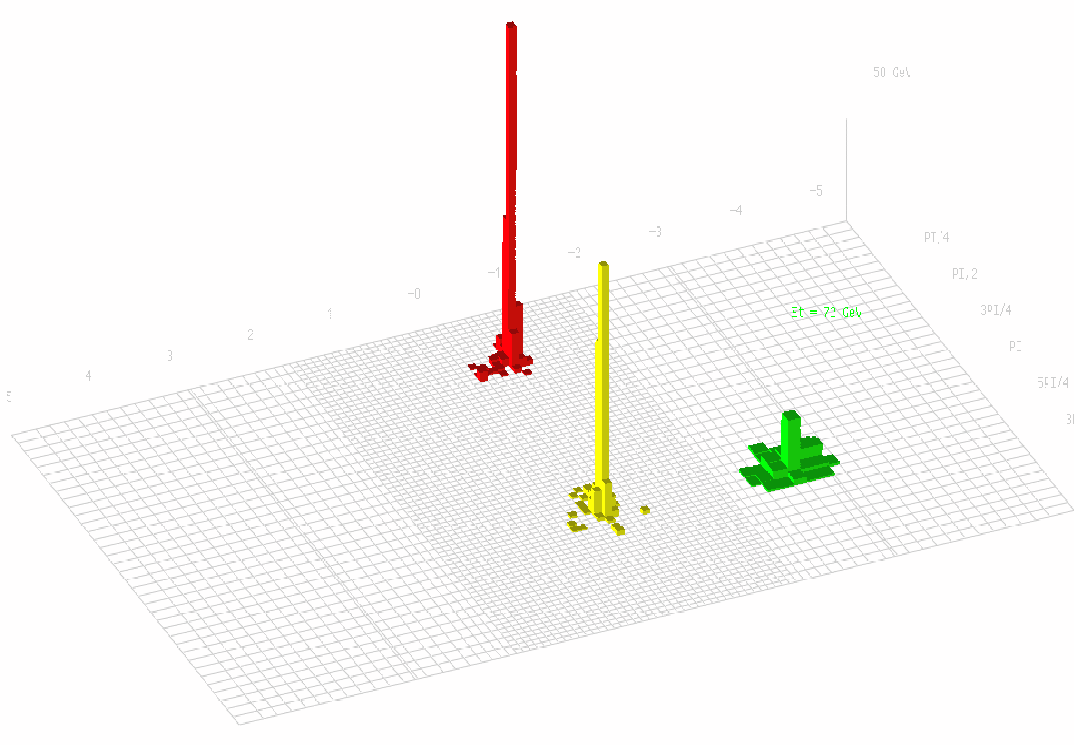

Figure 1: The $\eta, \varphi$ tower segmentation in CMS. The towers are defined to match the granularity of the hadron calorimeter.

The missing transverse energy vector is calculated by summing individual calorimeter towers having energy $E_{n}$, pseudorapidity $\eta_{n}$ and azimuthal angle $\varphi_{n}$ : 


$$
\mathbf{E}_{\mathrm{T}}^{\mathrm{miss}}=-\Sigma\left(\frac{E_{n} \cos \varphi_{n}}{\cosh \eta_{n}} \hat{\mathbf{x}}+\frac{E_{n} \sin \varphi_{n}}{\cosh \eta_{n}} \hat{\mathbf{y}}\right)=E_{x}^{\mathrm{miss}} \hat{\mathbf{x}}+E_{y}^{\mathrm{miss}} \hat{\mathbf{y}}
$$

Reconstructed muons are taken into account by replacing the expected calorimeter deposit (about $4 \mathrm{GeV}$ ) with the reconstructed track $p_{\mathrm{T}}$. Section 8 discusses further corrections to the reconstructed missing transverse energy.

\section{Expected Performance in Minimum-Bias Collisions}

Accurate measurement of the missing transverse energy in individual events is a difficult experimental problem, because various detector factors are known to contribute in subtle ways. These factors include energy resolution, limited detector coverage, nonlinearity of calorimeter response, detector granularity, non-instrumented material, magnetic field and its effect on low $p_{\mathrm{T}}$ charged particles, quantization of detector readout, electronic noise, event pileup, and underlying event. In spite of all these detector subtleties, the $\mathbf{E}_{\mathrm{T}}^{\mathrm{miss}}$ resolution in CMS is expected to be dominated by calorimeter energy resolution.

The total scalar transverse energy $\left(\Sigma E_{\mathrm{T}}\right)$, defined as the scalar $E_{\mathrm{T}}$ sum of all calorimeter towers in an event, is a quantity highly associated with $E_{\mathrm{T}}^{\text {miss }}$ [12]. Many properties of reconstructed missing transverse energy can be expressed as a function of $\Sigma E_{\mathrm{T}}$ because of its direct influence on the $E_{\mathrm{T}}^{\text {miss }}$ resolution via stochastic effects of calorimeter showers and the process of signal collection. The missing transverse energy resolution is normally determined by fitting the width of the measured $E_{x}^{\text {miss }}$ or $E_{y}^{\text {miss }}$ distribution in a sample of events in which no missing transverse energy is expected [5]. In minimum bias collisions at UA1, the resolution was observed to follow the form $\sigma=0.4 \sqrt{\Sigma E_{\mathrm{T}}} \mathrm{GeV}^{1 / 2}$ where the constant 0.4 depends directly on the stochastic term $0.8 \sqrt{E_{\mathrm{T}}} \mathrm{GeV}^{1 / 2}$ in the calorimeter resolution [5],[13]. The CDF experiment, which has a scintillating tile geometry similar to CMS (and a completely different magnetic field configuration compared to UA1, solenoid $v s$. dipole), observed a transverse energy resolution of $\sigma=0.47 \sqrt{\Sigma E_{\mathrm{T}}} \mathrm{GeV}^{1 / 2}$ in Run I [14]. From the UA1 and CDF results and the measured CMS calorimeter resolution stochastic term from test beam of $1.15 \sqrt{E_{\mathrm{T}}} \mathrm{GeV}^{1 / 2}$, one may expect a missing transverse resolution in CMS of $\sigma \approx(0.6-0.7) \sqrt{\Sigma E_{\mathrm{T}}} \mathrm{GeV}^{1 / 2}$ for minimum bias events with no pileup, when dominated by the shower fluctuations.

\section{Event Samples}

Previous studies of $E_{\mathrm{T}}^{\mathrm{miss}}$ in CMS [15] were limited by the use of less sophisticated simulation and reconstruction tools. A more advanced understanding of $E_{\mathrm{T}}^{\text {miss }}$ in the CMS detector has required the large event samples available from the recent data challenge [16]. These Monte Carlo events with full-detector simulation have been used to study the $E_{\mathrm{T}}^{\mathrm{miss}}$ performance of the CMS detector and to develop correction techniques, as well as to evaluate and optimize the trigger [17].

The samples were generated with PYTHIA [18] using the CMS software package CMKIN 3.0.0 [19], simulated with Geant4 [20] using the CMS software package OSCAR 2.4.5 [21], and digitized with the CMS objectoriented reconstruction code ORCA 7.6.1 [22]. The signal events are combined with low-luminosity $(\mathcal{L}=$ $2 \times 10^{33} \mathrm{~cm}^{-2} \mathrm{~s}^{-1}$ ) pileup corresponding to an average of 3.4 fully inelastic collisions per $25 \mathrm{~ns}$ beam crossing. The following samples were used in this study: $10^{6}$ minimum bias events, $3.2 \times 10^{6} \mathrm{QCD}$ events with parton transverse momenta $0<\hat{p}_{\mathrm{T}}<4000 \mathrm{GeV} / c, 5 \times 10^{5} \mathrm{~W}+$ jet events, and $4 \times 10^{5} \mathrm{t} \overline{\mathrm{t}}$ events. Details about the data samples, generation and simulation parameters, and reconstruction description can be found in ref. [24].

Event reconstruction and physics analysis was performed with ORCA 8.7.1 [22]. No additional thresholds on the calorimeter towers, beyond the noise suppression cuts already mentioned, are applied for jet reconstruction or the $E_{\mathrm{T}}^{\mathrm{miss}}$ calculation. Muons reconstructed at level-3 (offline) [15] are included in the determination of $E_{\mathrm{T}}^{\mathrm{miss}}$. No significant difference is observed in the $E_{\mathrm{T}}^{\mathrm{miss}}$ resolution for the QCD samples between two different muon algorithms used in ORCA [23].

\section{Performance in Soft Collisions}

\subsection{Minimum Bias Without Pileup}

Figure 2(a) shows the distribution of reconstructed $\Sigma E_{\mathrm{T}}$ from a high-statistics sample of fully-simulated minimum bias events without pileup. The event simulation includes single and double diffraction. There is a minimum value of reconstructed $\Sigma E_{\mathrm{T}}$, an offset, that is dependent on the calorimeter cell thresholds, corresponding to an online zero-suppression, that are used to form the calorimeter towers. For this sample, the offset is $142 \mathrm{GeV}$. The average 
value of $194 \mathrm{GeV}$ corresponds to $\Sigma E_{\mathrm{T}}$ of $52 \mathrm{GeV}$ above the offset. This $52 \mathrm{GeV}$ of event activity determines the size of the stochastic term in the $E_{\mathrm{T}}^{\mathrm{miss}}$ resolution for this sample. Figure 2(b) shows the distribution of $E_{x}^{\text {miss }}$. The width of this distribution is $\sigma=6.1 \mathrm{GeV}$ in agreement with expectation of addition in quadrature of a 3.8 $\mathrm{GeV}$ noise term and a $0.63 \sqrt{\Sigma E_{\mathrm{T}}} \mathrm{GeV}^{1 / 2}$ stochastic term. It is important to note that at this stage, the vector $\mathbf{E}_{\mathrm{T}}^{\text {miss }}=E_{x} \hat{\mathbf{x}}+E_{y} \hat{\mathbf{y}}$ is made using towers that have been formed from ECAL cells calibrated for photons and HCAL cells calibration for hadrons, appropriate perhaps for understanding the detector response to first collisions. It is believed that making use of energy flow techniques such as the charged track corrections [25] will ultimately improve the $E_{\mathrm{T}}^{\text {miss }}$ resolution.

(a)

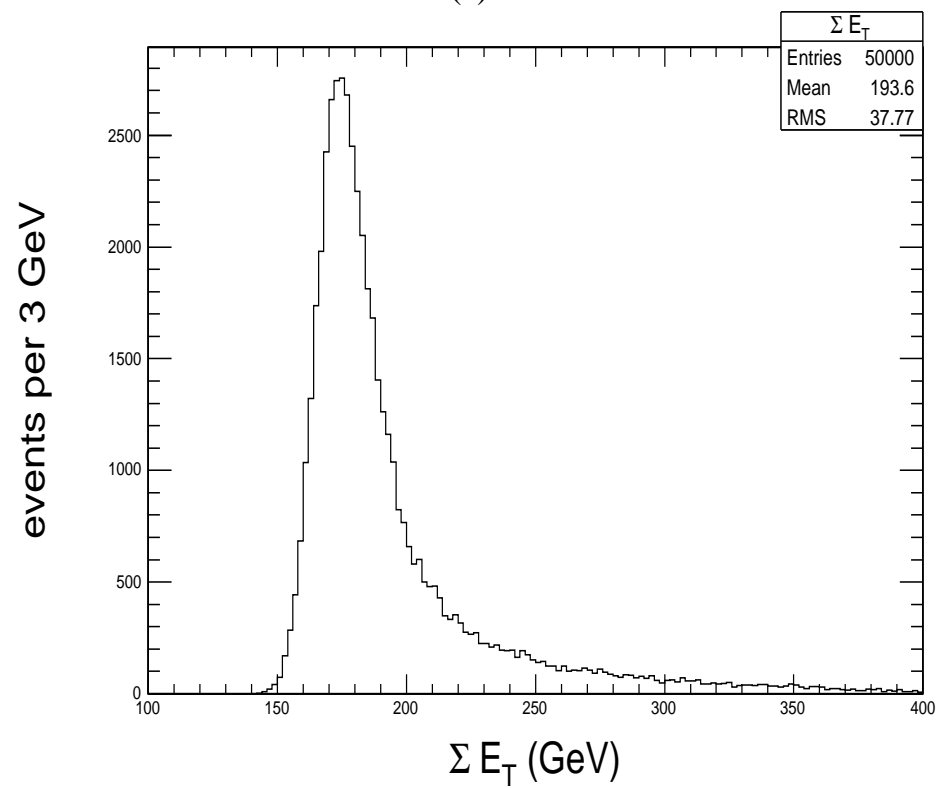

(b)

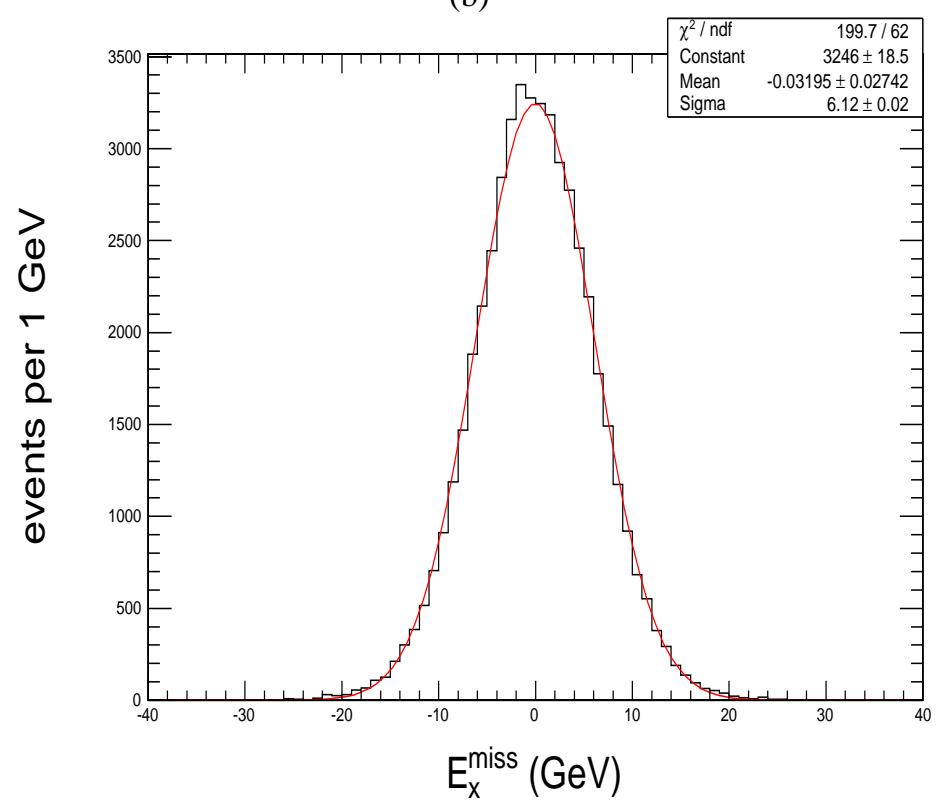

Figure 2: (a) $\Sigma E_{\mathrm{T}}$ for minimum bias events with no pileup. The reconstructed offset (minimum value of $\Sigma E_{\mathrm{T}}$ ) of $142 \mathrm{GeV}$ depends on calorimeter thresholds. (b) Distribution of $E_{x}^{\mathrm{miss}}$ in the same events. The resolution is fit to a Gaussian with $\sigma=6.1 \mathrm{GeV}$, in agreement with expectations based on a stochastic term of $0.63 \sqrt{\Sigma E_{\mathrm{T}}} \mathrm{GeV}^{1 / 2}$ and a noise contribution of $3.8 \mathrm{GeV}$. 


\subsection{Soft Collisions With Pileup}

The addition of pileup increases the observed $\Sigma E_{\mathrm{T}}$ as shown in Fig. 3(a) for a sample of soft QCD collisions with $0<\hat{p}_{\mathrm{T}}<15 \mathrm{GeV} / c$. The average value of $\Sigma E_{\mathrm{T}}$ in this sample is $166 \mathrm{GeV}$ greater than that of the minimum bias sample without pileup (Fig. 2(a)). The corresponding missing transverse energy resolution shown in Fig. 3(b) is $9.9 \mathrm{GeV}$. This increase from $6.1 \mathrm{GeV}$ (without pileup) to $9.9 \mathrm{GeV}$ (with pileup) is in agreement with expectations from a stochastic term of $0.63 \sqrt{\Sigma E_{\mathrm{T}}} \mathrm{GeV}^{1 / 2}$. Thus, the presence of pileup does not adversely affect the $E_{\mathrm{T}}^{\text {miss }}$ resolution beyond raising the overall activity $\left(\Sigma E_{\mathrm{T}}\right)$ of the event.

(a)

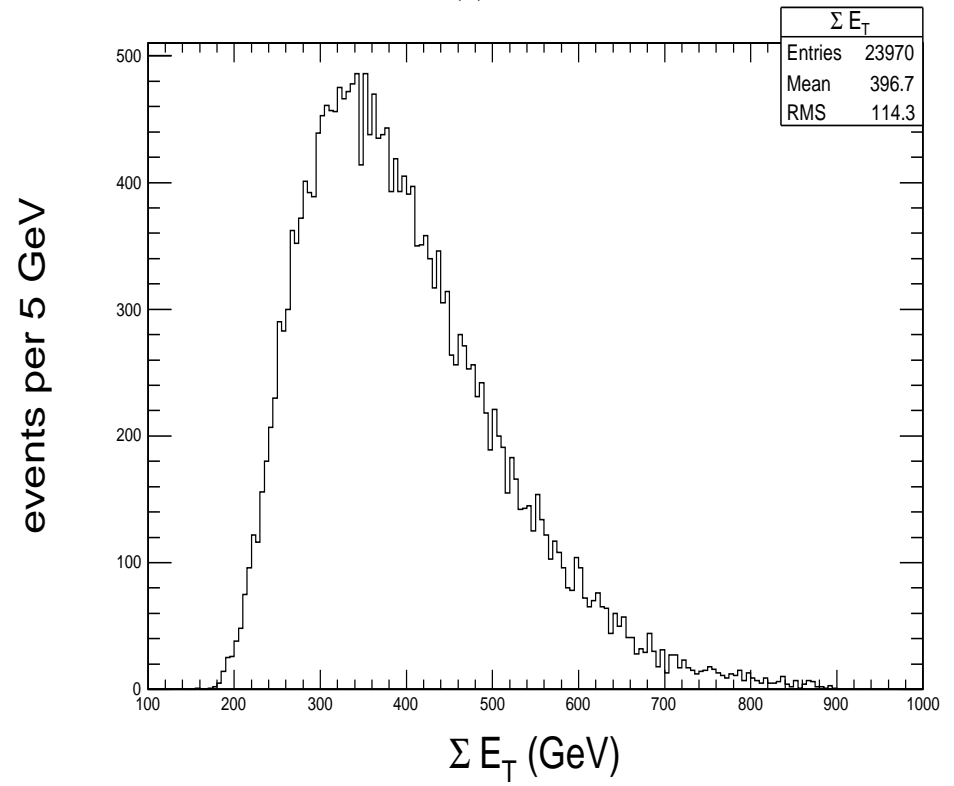

(b)

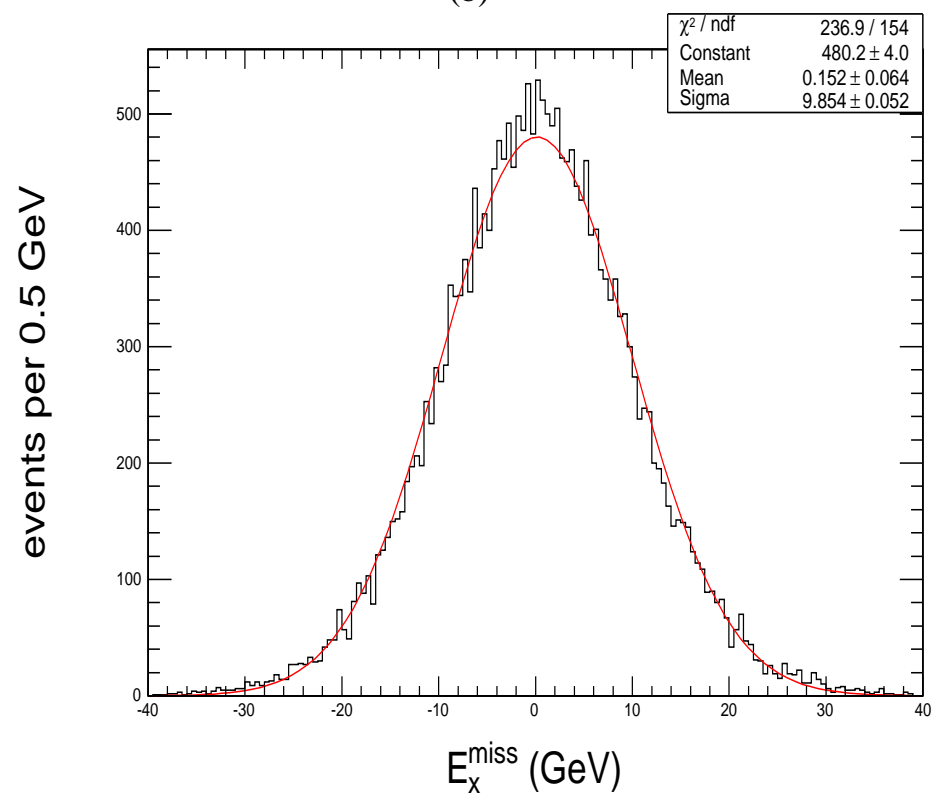

Figure 3: (a) Distribution of $\Sigma E_{\mathrm{T}}$ for soft QCD collisions $\left(0<\hat{p}_{\mathrm{T}}<15 \mathrm{GeV} / c\right)$, including event pileup corresponding to a luminosity of $\mathcal{L}=2 \times 10^{33} \mathrm{~cm}^{-2} \mathrm{~s}^{-1}$. (b) Distribution of $E_{x}^{\text {miss }}$ for the same events. The resolution is fit to a Gaussian with $\sigma=9.9 \mathrm{GeV}$.

A more detailed comparison of the reconstructed missing transverse energy resolution from minimum bias events and soft QCD events is shown in Fig. 4. The minimum bias and QCD samples of Fig. 4 both include event pileup corresponding to a luminosity of $\mathcal{L}=2 \times 10^{33} \mathrm{~cm}^{-2} \mathrm{~s}^{-1}$. Figure 4 shows the $E_{\mathrm{T}}^{\text {miss }}$ resolution, determined from fits to reconstructed $E_{x}^{\text {miss }}$ distributions like that of Fig. 3(b), for minimum bias events (open circles) and soft QCD 
events, $0<\hat{p}_{\mathrm{T}}<15 \mathrm{GeV} / c$, (solid squares). The resolution as a function of $\Sigma E_{\mathrm{T}}$ has parameterized with a fit (solid curve in Fig. 4) that gives

$$
\sigma=\left[(4.0 \mathrm{GeV})^{2}+\left(0.63 \mathrm{GeV}^{1 / 2} \sqrt{\Sigma E_{\mathrm{T}}-142 \mathrm{GeV}}\right)^{2}\right]^{1 / 2} .
$$

The stochastic term is the same as observed in minimum bias collisions with no pileup (Fig, 2(b)).

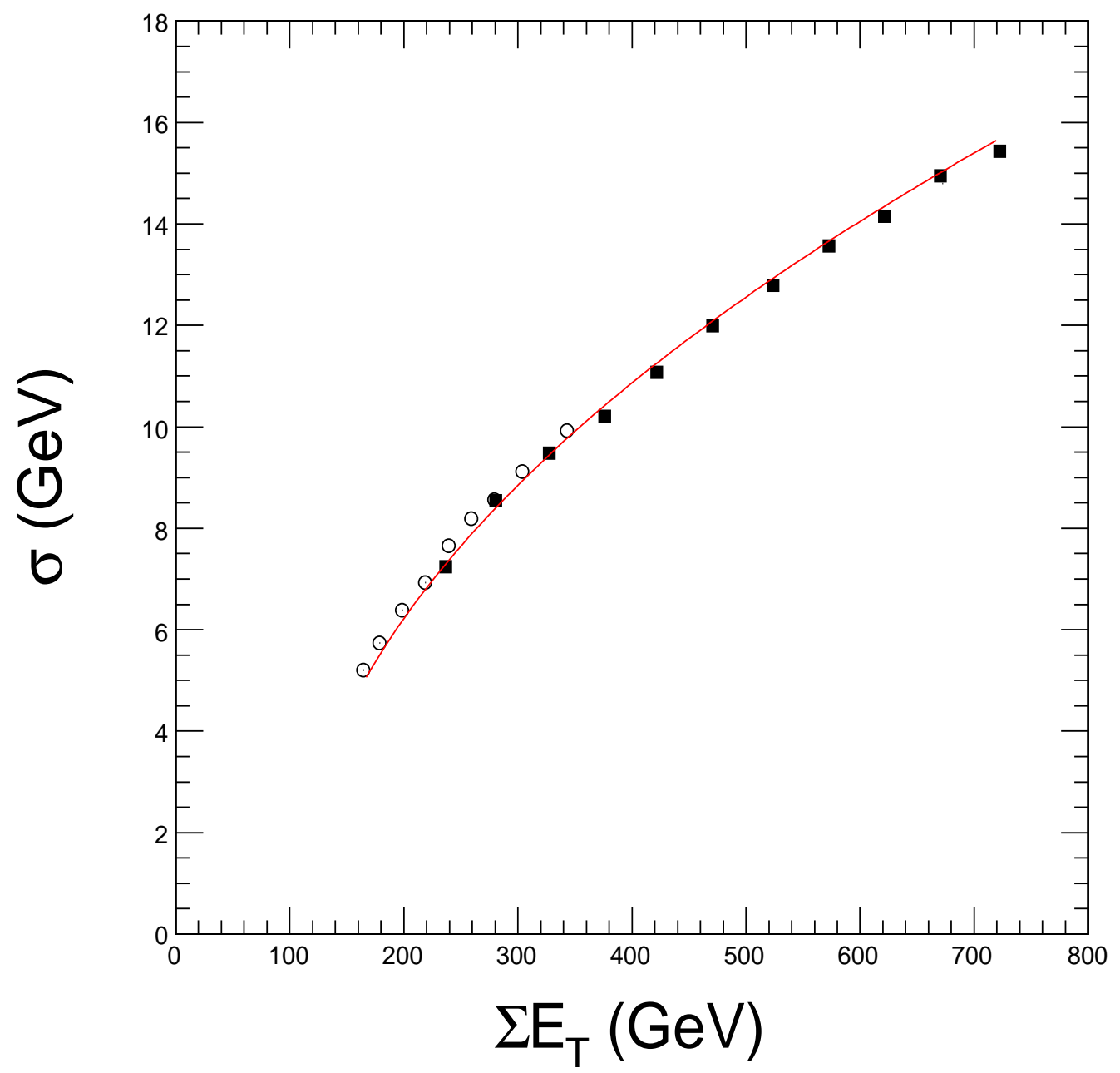

Figure 4: Missing transverse energy resolution, as determined from Gaussian fits to distributions of reconstructed $E_{x}^{\text {miss }}, v s$. reconstructed $\Sigma E_{\mathrm{T}}$ for soft QCD events, $0<\hat{p}_{\mathrm{T}}<15 \mathrm{GeV} / c$, (solid squares) and minimum bias events (open circles), including event pileup corresponding to a luminosity of $\mathcal{L}=2 \times 10^{33} \mathrm{~cm}^{-2} \mathrm{~s}^{-1}$. The resolution (curve) has been fit to $\sigma=\left[(4.0 \mathrm{GeV})^{2}+\left(0.63 \mathrm{GeV}^{1 / 2} \sqrt{\Sigma E_{\mathrm{T}}-142 \mathrm{GeV}}\right)^{2}\right]^{1 / 2}$.

\section{Performance in Hard Collisions}

\subsection{Reconstructed Missing Transverse Energy}

For intrinsically balanced 2-to-2 scattering processes, the reconstructed $E_{\mathrm{T}}^{\text {miss }}$ is directly related to the $E_{\mathrm{T}}^{\text {miss }}$ resolution [6]. It is well known that missing transverse energy resolution measured in high momentum-transfer events will not fit the same stochastic term as soft collision. This is due in part to fragmentation effects and contributions to the jet resolution from linear terms [26]. The UA1 experiment $(\sqrt{s}=0.54 \mathrm{TeV})$ measured a missing transverse energy, $E_{\mathrm{T}}^{\text {miss }}=\sqrt{\left(E_{x}^{\text {miss }}\right)^{2}+\left(E_{y}^{\text {miss }}\right)^{2}}$, in jet events corresponding to a stochastic coefficient of 0.7 [6], while the CDF experiment $(\sqrt{s}=1.8 \mathrm{TeV})$ determined a stochastic coefficient near unity [27]. From these results, we may expect the distribution of $E_{\mathrm{T}}^{\mathrm{miss}}$ in jet events for CMS to fit a stochastic coefficient in the range 1.1-1.3. 
Figure 5 shows (a) the reconstructed $\Sigma E_{\mathrm{T}}$ and (b) $E_{\mathrm{T}}^{\text {miss }}$ for QCD events generated with $80 \mathrm{GeV} / c<\hat{p}_{\mathrm{T}}<$ $120 \mathrm{GeV} / c$. The observed $E_{\mathrm{T}}^{\text {miss }}$ distribution, by its construction, has a one-sided tail. A small $\hat{p}_{\mathrm{T}}$ interval can create a wide spectrum of $E_{\mathrm{T}}^{\text {miss }}$ at both generator and detector level. The reconstructed $E_{\mathrm{T}}^{\text {miss }}$ extends to large values $(>50 \mathrm{GeV})$ due to a small number of events containing one or more energetic neutrinos or non-identified muons from heavy flavor ( $b$ and $c$ ) decays.

(a)

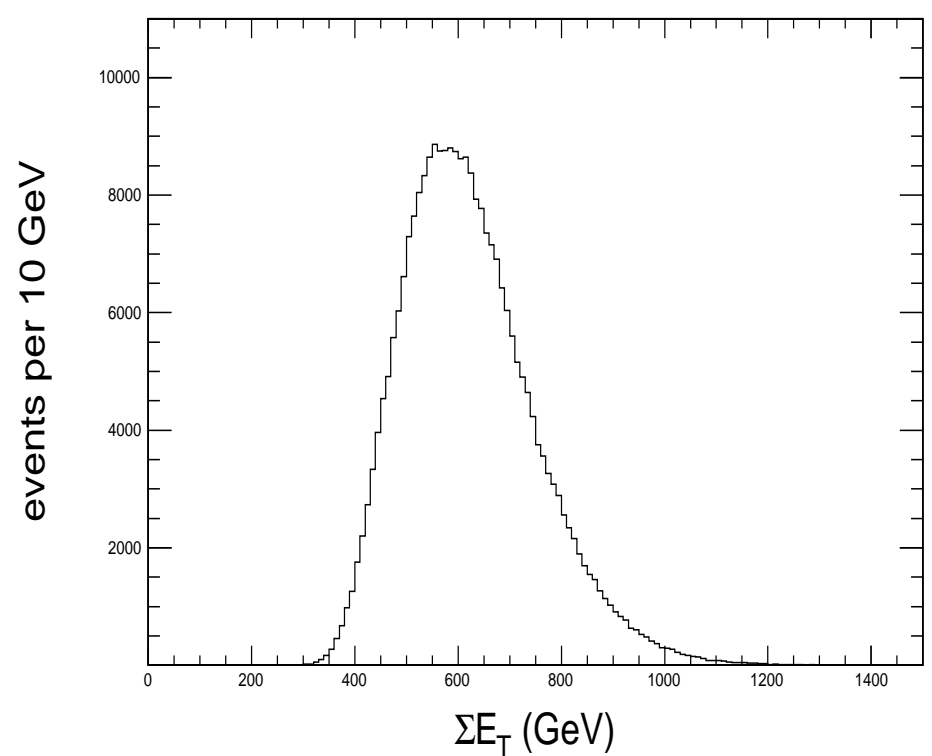

(b)

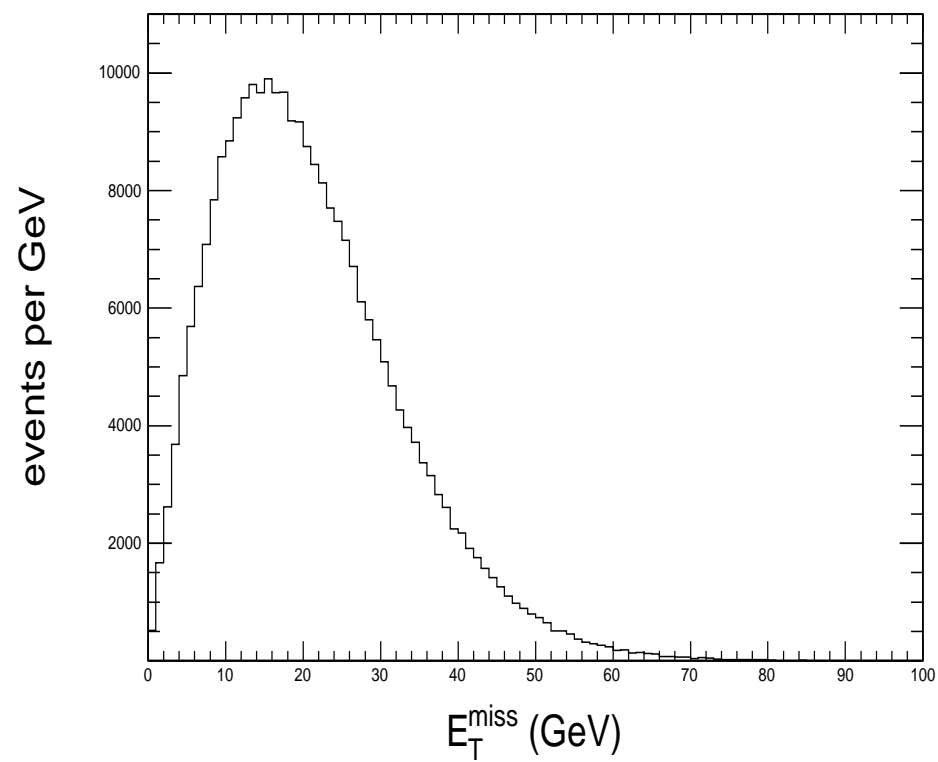

Figure 5: (a) Reconstructed $\Sigma E_{\mathrm{T}}$ in a QCD sample with $80 \mathrm{GeV} / c<\hat{p}_{\mathrm{T}}<120 \mathrm{GeV} / c$. The sample includes pileup corresponding to a luminosity of $\mathcal{L}=2 \times 10^{33} \mathrm{~cm}^{-2} \mathrm{~s}^{-1}$. (b) Reconstructed $E_{\mathrm{T}}^{\text {miss }}$ in the same sample.

The average reconstructed $E_{\mathrm{T}}^{\text {miss }}$ in QCD events (with pileup) as a function of $\Sigma E_{\mathrm{T}}$ is shown in Fig. 6. At $\Sigma E_{\mathrm{T}}$ $=2100 \mathrm{GeV}$, which corresponds to jets with reconstructed $p_{\mathrm{T}} \approx 800 \mathrm{GeV} / c$, an average $E_{\mathrm{T}}^{\text {miss }}$ of about $60 \mathrm{GeV}$ is reconstructed. This number is consistent with measurement of such jets with a resolution of $1.25 \mathrm{GeV}^{1 / 2} \sqrt{E_{\mathrm{T}}}[28]$ plus a small contribution from the underlying event. Due to the increased occupancy in these events, the offset from noise contributions in the reconstructed $\Sigma E_{\mathrm{T}}$ distribution has shifted up to $350 \mathrm{GeV}$ as shown in Fig. 5(a). A fit to the reconstructed missing transverse energy (curve in Fig. 6) gives

$$
\left\langle E_{\mathrm{T}}^{\text {miss }}\right\rangle=\left[(5.4 \mathrm{GeV})^{2}+\left(1.23 \mathrm{GeV}^{1 / 2} \sqrt{\Sigma E_{\mathrm{T}}-350 \mathrm{GeV}}\right)^{2}+\left[0.019\left(\Sigma E_{\mathrm{T}}-350 \mathrm{GeV}\right)\right]^{2}\right]^{1 / 2}
$$


where the offset in $\Sigma E_{\mathrm{T}}$ is subtracted as shown. This result is consistent with measurements from UA1 and CDF as noted above.

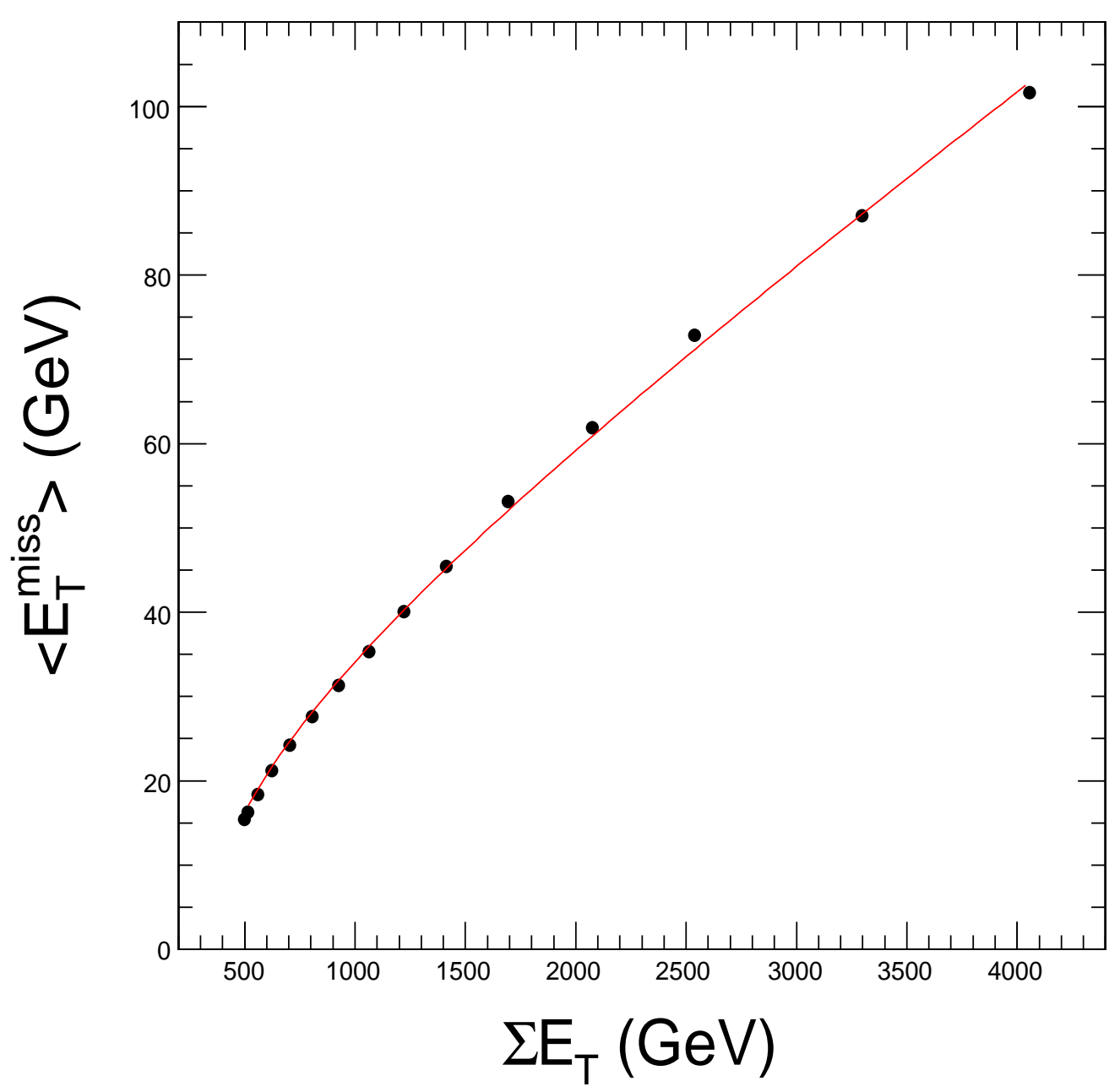

Figure 6: Average reconstructed $E_{\mathrm{T}}^{\mathrm{miss}}$ vs. $\Sigma E_{\mathrm{T}}$ for QCD hard collisions, including event pileup corresponding to a luminosity of $\mathcal{L}=2 \times 10^{33} \mathrm{~cm}^{-2} \mathrm{~s}^{-1}$. The fit (curve) corresponds to $\left\langle E_{\mathrm{T}}^{\text {miss }}\right\rangle=\left[(5.4 \mathrm{GeV})^{2}+\right.$ $\left.\left(1.23 \mathrm{GeV}^{1 / 2} \sqrt{\Sigma E_{\mathrm{T}}-350 \mathrm{GeV}}\right)^{2}+\left[0.019\left(\Sigma E_{\mathrm{T}}-350 \mathrm{GeV}\right)\right]^{2}\right]^{1 / 2}$.

\subsection{Scalar Transverse Energy Sum}

The distribution of $\Sigma E_{\mathrm{T}}$ was investigated for QCD collisions with varying values of generator $\hat{p}_{\mathrm{T}}$ from 20-1000 $\mathrm{GeV}$, including the effects of pileup corresponding to a luminosity of $\mathcal{L}=2 \times 10^{33} \mathrm{~cm}^{-2} \mathrm{~s}^{-1}$. As noted above, a relatively small $\hat{p}_{\mathrm{T}}$ interval corresponds to a wide range of $\Sigma E_{\mathrm{T}}$ (see Fig. 5). Figure 7 shows the average reconstructed $E_{\mathrm{T}}$ of the leading jets $v s$. reconstructed $\left(\Sigma E_{\mathrm{T}}\right)_{\mathrm{r}}$. The points correspond to parton-level $\hat{p}_{\mathrm{T}}$ ranges of (from left to right) of 20-30, 30-50, 50-80, 80-120, 120-170, 170-230, 230-300, 300-380, 380-470, 470-600, 600-800 and 800-1000 GeV/c. This allows us to interpret the $\Sigma E_{\mathrm{T}}$ scale (e.g., Fig. 6) in terms of the dominating jet activity.

Figure 8 shows the ratio of reconstructed $\left(\Sigma E_{\mathrm{T}}\right)_{\mathrm{r}}$ to generated $\left(\Sigma E_{\mathrm{T}}\right)_{\mathrm{g}}$ summed transverse energy $v s .\left(\Sigma E_{\mathrm{T}}\right)_{\mathrm{g}}$. The larger value of $\left(\Sigma E_{\mathrm{T}}\right)_{\mathrm{r}}$ compared to $\left(\Sigma E_{\mathrm{T}}\right)_{\mathrm{g}}$ at small values of $\left(\Sigma E_{\mathrm{T}}\right)_{\mathrm{g}}$ is due to a threshold-dependent offset in the reconstructed $\Sigma E_{\mathrm{T}}$ scale due to electronic noise.

Figure 9 shows the contribution of pileup to the reconstructed $\left(\Sigma E_{\mathrm{T}}\right) v s$. generated $\left(\Sigma E_{\mathrm{T}}\right)$ in QCD events. The contribution of pileup to the reconstructed $\left(\Sigma E_{\mathrm{T}}\right)$ is about $350-450 \mathrm{GeV}$. The dependence of the pileup contribution on the generated (signal) $\Sigma E_{\mathrm{T}}$ comes from various detector effects including nonlinear response, electronic noise, and the $E_{\mathrm{T}}$ threshold used in calorimeter hit reconstruction $(0.5 \mathrm{GeV}$ for HCAL). 


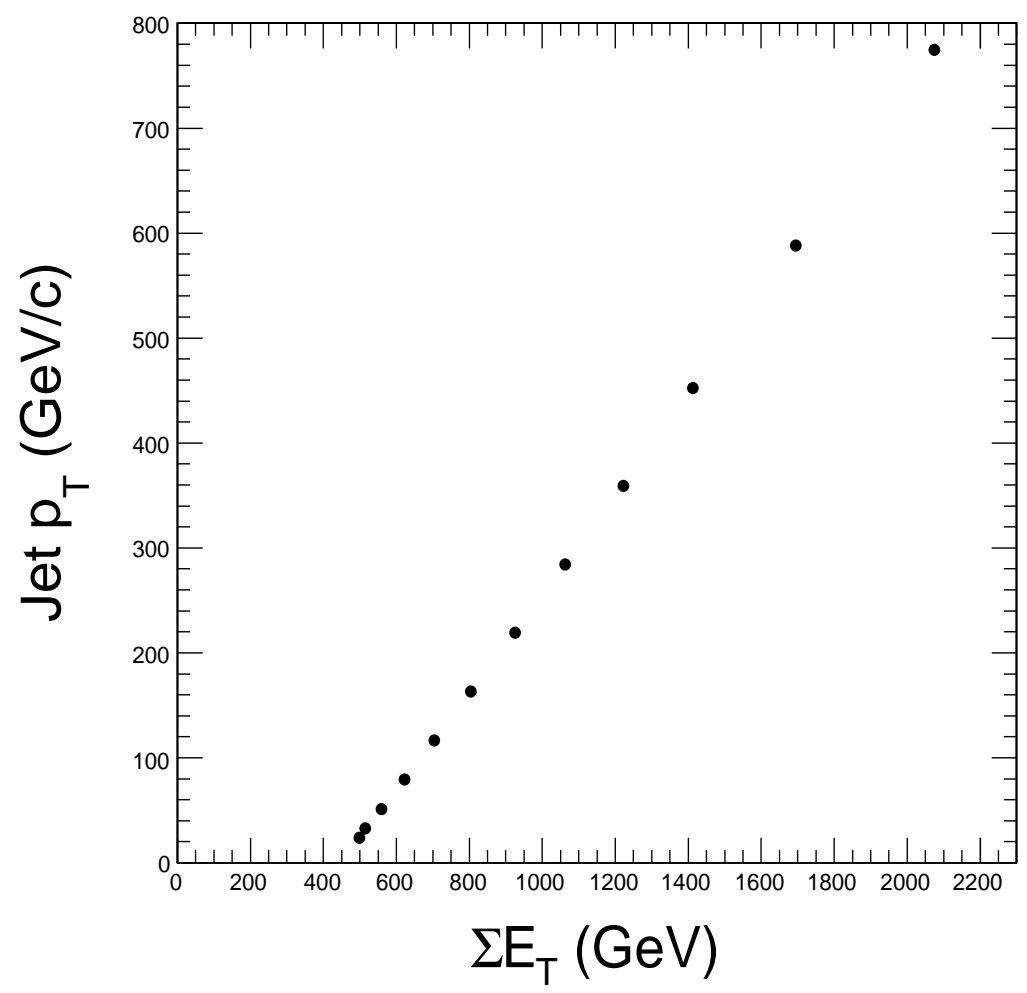

Figure 7: Average reconstructed $p_{\mathrm{T}}$ of the leading jets $v s$. reconstructed $\Sigma E_{\mathrm{T}}$. The points correspond to partonlevel $\hat{p}_{\mathrm{T}}$ ranges of (from left to right) of 20-30, 30-50, 50-80, 80-120, 120-170, 170-230, 230-300, 300-380, $380-470,470-600,600-800$ and 800-1000 GeV/c.

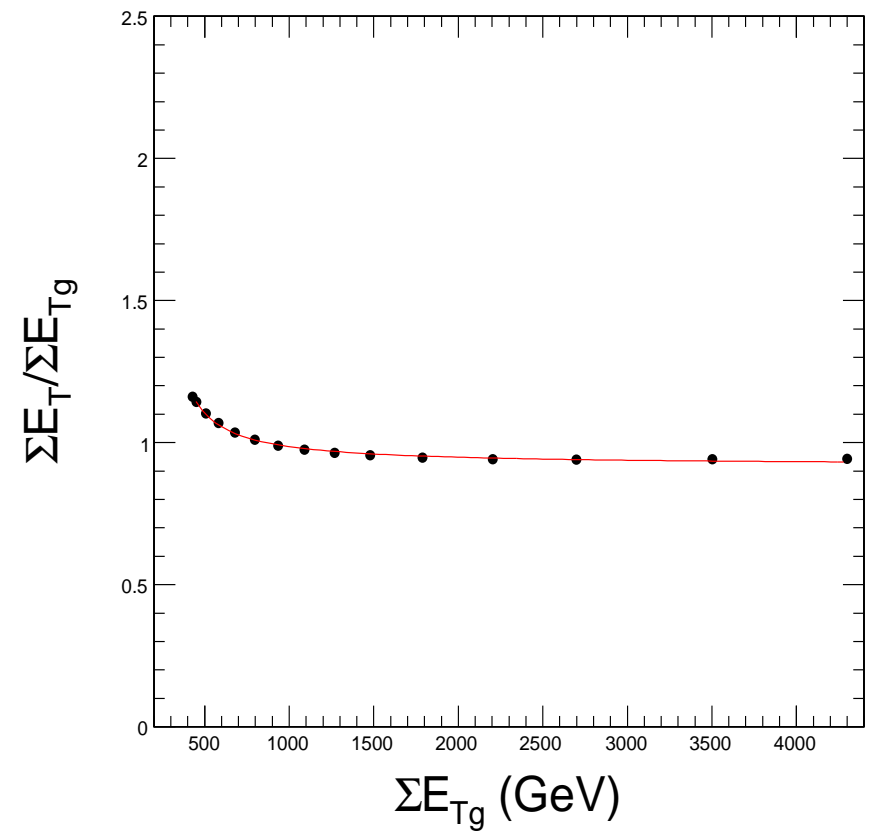

Figure 8: Ratio of reconstructed $\left(\Sigma E_{\mathrm{T}}\right)_{\mathrm{r}}$ to generated $\left(\Sigma E_{\mathrm{T}}\right)_{\mathrm{g}}$ summed transverse energy $v s .\left(\Sigma E_{\mathrm{T}}\right)_{\mathrm{g}}$ from QCD events with pileup. The increase in the ratio at low values of $\left(\Sigma E_{\mathrm{T}}\right)_{\mathrm{g}}$ is due to a threshold-dependent offset in $\left(\Sigma E_{\mathrm{T}}\right)_{\mathrm{r}}$ caused by electronic noise. The ratio is fit (curve) to $\left(\Sigma E_{\mathrm{T}}\right)_{\mathrm{r}} /\left(\Sigma E_{\mathrm{T}}\right)_{\mathrm{g}}=0.92+240 \mathrm{GeV} /\left[\left(\Sigma E_{\mathrm{T}}\right)_{\mathrm{g}}-\right.$ $40 \mathrm{GeV}]$. 


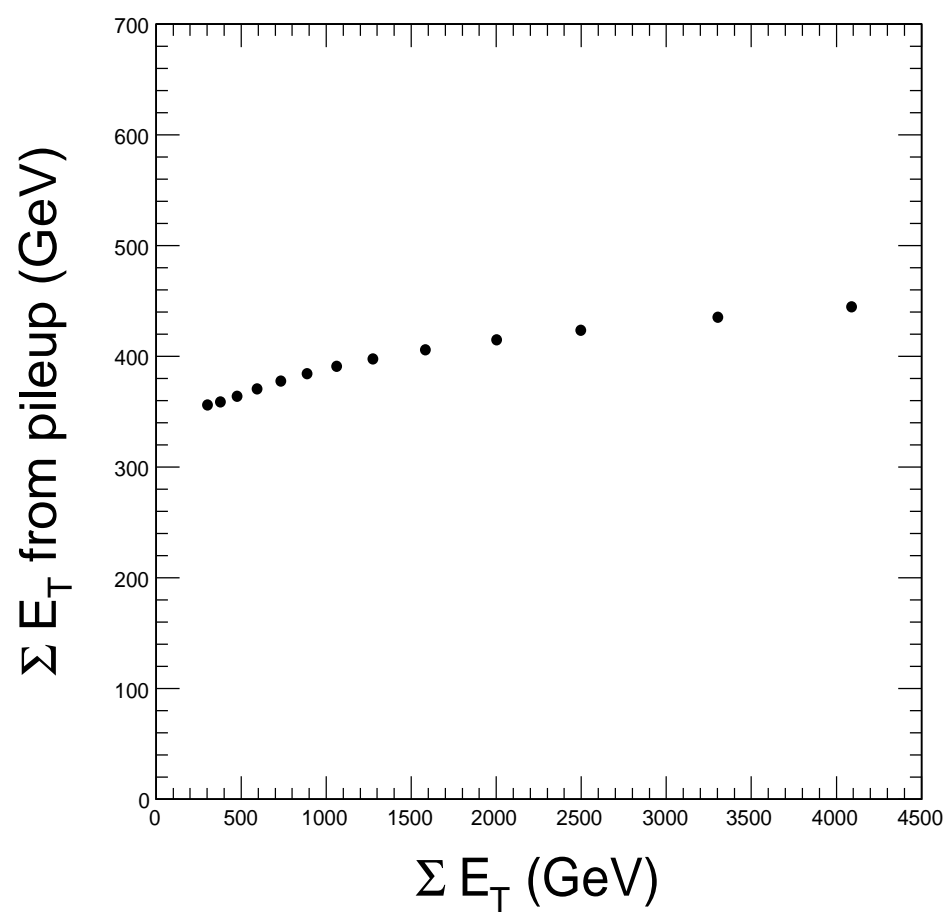

Figure 9: Contribution of pileup to reconstructed $\Sigma E_{\mathrm{T}}$ as a function of generated $\Sigma E_{\mathrm{T}}$. The slight dependence on generated $\Sigma E_{\mathrm{T}}$ is due to detector effects.

\subsection{Missing Transverse Energy Resolution}

Figure 10 shows the $E_{\mathrm{T}}^{\mathrm{miss}}$ resolution, determined from fits to reconstructed $E_{x}^{\mathrm{miss}}$ distributions, for QCD hard collisions, including event pileup corresponding to a luminosity of $\mathcal{L}=2 \times 10^{33} \mathrm{~cm}^{-2} \mathrm{~s}^{-1}$. At low values of $\Sigma E_{\mathrm{T}}$, the resolution (Fig. 10) agrees with that determined from soft collisions (Fig. 4). The resolution as a function of $\Sigma E_{\mathrm{T}}$ is parameterized with a fit (curve in Fig. 10) that gives

$$
\sigma=\left[(3.8 \mathrm{GeV})^{2}+\left(0.97 \mathrm{GeV}^{1 / 2} \sqrt{\Sigma E_{\mathrm{T}}-350 \mathrm{GeV}}\right)^{2}+\left[0.012\left(\Sigma E_{\mathrm{T}}-350 \mathrm{GeV}\right)\right]^{2}\right]^{1 / 2} .
$$

This result is consistent with that measured in UA1 [6] and CDF [27] when scaled by the measured performance of the calorimetry in test beams. The large QCD dijet cross section at the LHC will allow us to measure distributions of $E_{x}^{\text {miss }}$ and $E_{y}^{\text {miss }}$ as a function of instantaneous luminosity in order to directly determine the missing transverse energy resolution under operating conditions.

\section{Contribution of Jets and Unclustered Towers}

Since jets dominate the $E_{\mathrm{T}}$ contribution in QCD events, it is interesting to investigate out-of-cone radiation and underlying event effects by separating the contributions of reconstructed jets and unclustered towers to the reconstructed missing transverse energy. Two regions are defined in an event: the jet region (defined by the jet cones) and the unclustered region (all calorimeter towers that are outside jets). The value of $E_{\mathrm{T}}^{\text {miss }}$ can be calculated separately for each region. The two regions are defined by the reconstructed event at the detector level; at the generator level, particles are associated with one of the two regions based on their direction. The definition of regions is performed for every event.

Jets are reconstructed by the iterative cone (IC) algorithm implemented in ORCA with cone size of $R=0.5$ and a minimum jet threshold of $p_{\mathrm{T}}>20 \mathrm{GeV} / c$ [28]. The towers that do not contribute to jets are collected as unclustered towers. The jet cone size was varied to investigate the effect on the region definition and $E_{\mathrm{T}}^{\text {miss }}$ quantities. We use the axis of IC jets with a $R=0.5$ cone as input to rebuild the jets with new cone sizes of $R=0.2,0.4,0.6$ and 0.8. Fig. 11 shows the ratio of $\Sigma E_{\mathrm{T}}$ in the jet region to that in the unclustered region. as a function of reconstructed $\Sigma E_{\mathrm{T}}$ for the different cone sizes. A cone size of $R=0.2$ contains only the core of the jet [13]. At $\Sigma E_{\mathrm{T}}=1700 \mathrm{GeV}$ which corresponds to jets with average transverse momenta of approximately 530 


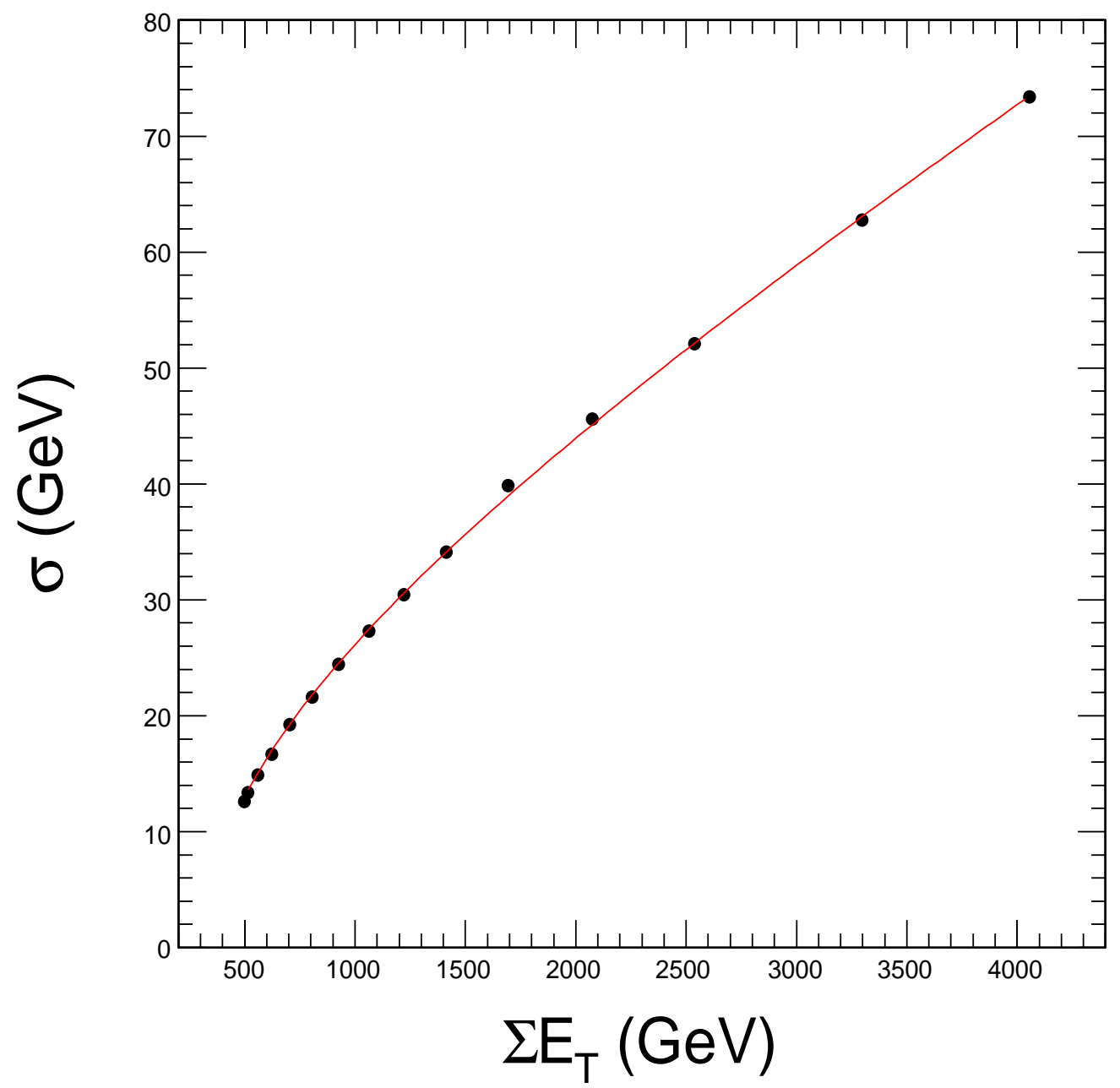

Figure 10: Missing transverse energy resolution $v s . \Sigma E_{\mathrm{T}}$ for QCD hard collisions, including event pileup corresponding to a luminosity of $\mathcal{L}=2 \times 10^{33} \mathrm{~cm}^{-2} \mathrm{~s}^{-1}$. The resolution (curve) has been fit to $\sigma=\left[(3.8 \mathrm{GeV})^{2}+\right.$ $\left.\left(0.97 \mathrm{GeV}^{1 / 2} \sqrt{\Sigma E_{\mathrm{T}}-350 \mathrm{GeV}}\right)^{2}+\left[0.012\left(\Sigma E_{\mathrm{T}}-350 \mathrm{GeV}\right)\right]^{2}\right]^{1 / 2}$

$\mathrm{GeV} / c$, about $3 / 4$ of the reconstructed $\Sigma E_{\mathrm{T}}$ is inside the $R=0.5$ jet cones. As expected, the jets play a dominant role in defining the reconstructed $E_{\mathrm{T}}^{\text {miss }}$ in the event. Figure 12 shows the contributions to the missing transverse energy resolution determined separately for the jet and unclustered regions.

For QCD events, it is expected that the direction of reconstructed missing transverse energy be opposite in $\varphi$ to that of the most energetic jet. Fig. 13 shows the $\varphi$ difference minus $\pi$ between the highest $E_{\mathrm{T}}$ jet and $\mathbf{E}_{\mathrm{T}}^{\text {miss }}$, indicating that the reconstructed $\mathbf{E}_{\mathrm{T}}^{\mathrm{miss}}$ vector tends to be opposite the leading jet in QCD events.

The direction ( $\varphi$ angle) of reconstructed missing transverse energy in the jet region is strongly correlated to that in the unclustered region indicating that the underlying event contributes to the overall balance of $E_{\mathrm{T}}^{\text {miss }}$. Figure 14 shows the distribution of $\Delta \varphi-\pi=\varphi_{\mathrm{j}}-\phi_{\mathrm{u}}-\pi$, where $\varphi_{\mathrm{j}}$ and $\varphi_{\mathrm{u}}$ are the azimuthal angles of the reconstructed missing transverse energy vectors in the jet and unclustered regions, for two different QCD samples, (a) $30 \mathrm{GeV} / c<\hat{p}_{\mathrm{T}}<50 \mathrm{GeV} / c$ and (b) $50 \mathrm{GeV} / c<\hat{p}_{\mathrm{T}}<80 \mathrm{GeV} / c$, and four different cone sizes $(R=0.2,0.4$, 0.6 , and 0.8 ). The correlation is stronger for lower $p_{\mathrm{T}}$ jets where the importance of the underlying event for overall $E_{\mathrm{T}}^{\text {miss }}$ balance is greater.

As an additional check of detector $\mathbf{E}_{\mathrm{T}}^{\mathrm{miss}}$ performance, one may look at the resolution in a direction orthogonal to the jet axis. By choosing this direction, the observed resolution is independent of the reconstructed jet resolution, but rather is dominated by the underlying event and pileup activity. This distribution is shown in Fig. 15 vs. reconstructed $\Sigma E_{\mathrm{T}}$. The result may be fit to the form (curve in Fig. 15) 


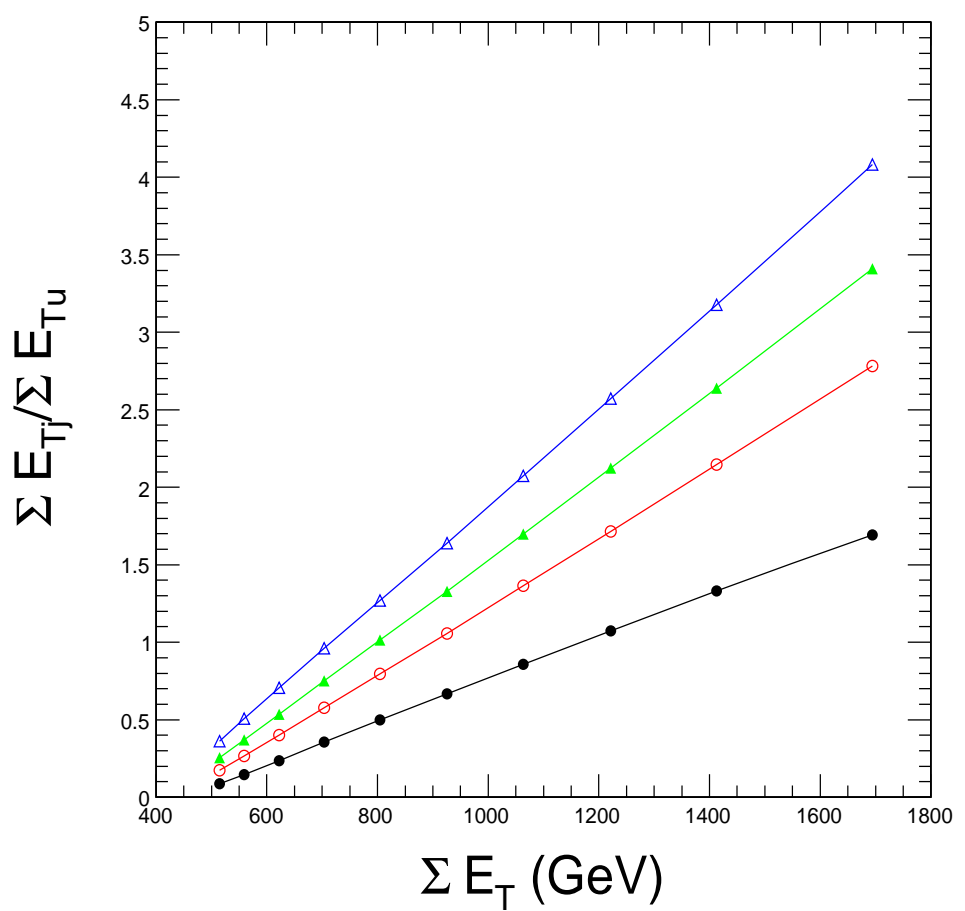

Figure 11: Ratio of reconstructed $\Sigma E_{\mathrm{T}}$ in the jet region to that in the unclustered region $v s . \Sigma E_{\mathrm{T}}$ for jet cones of size $R=0.2$ (solid circles), 0.4 (open circles), 0.6 (solid triangles), and 0.8 (open triangles).

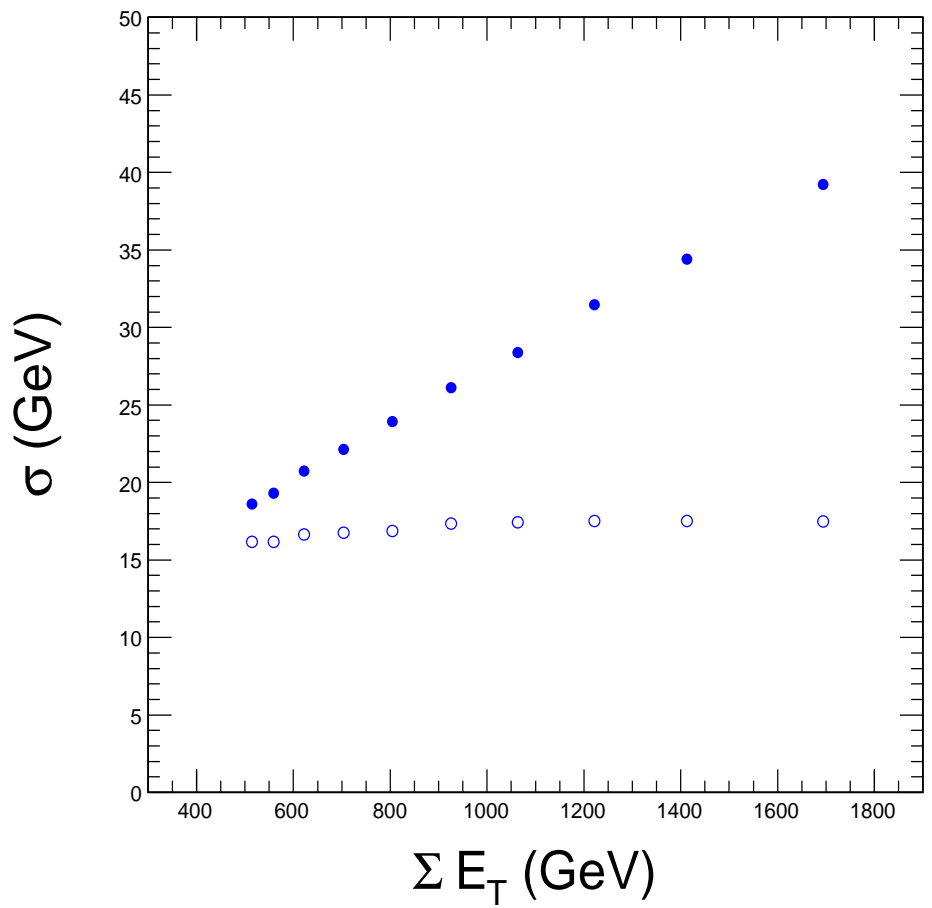

Figure 12: Contributions of deposits in the jet (solid circles) and unclustered regions (open circles) to the missing transverse energy resolution. $v s$. reconstructed $\Sigma E_{\mathrm{T}}$. 


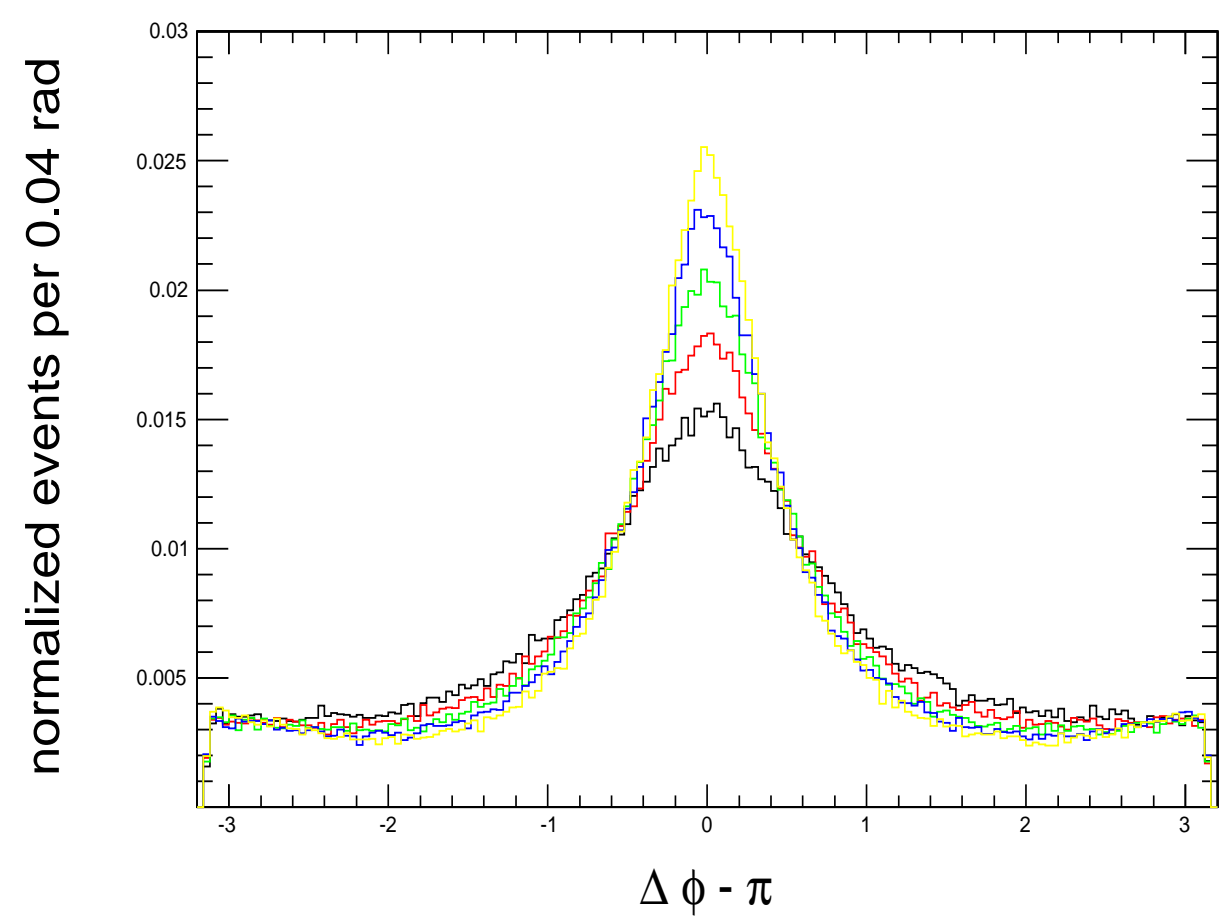

Figure 13: The $\varphi$ separation between the direction of the highest $E_{\mathrm{T}}$ jet and the reconstructed vector $\mathbf{E}_{\mathrm{T}}^{\mathrm{miss}}$. Five $\hat{p_{\mathrm{T}}}$ QCD samples are plotted: $50-80 \mathrm{GeV} / c$ (black), $80-120 \mathrm{GeV} / c$ (red), $120-170 \mathrm{GeV} / c$ (green), $170-230 \mathrm{GeV} / c$ (blue) and 230-300 GeV/c (yellow) GeV/c.

$$
\sigma=\left[(9.9 \mathrm{GeV})^{2}+\left(0.48 \mathrm{GeV}^{1 / 2} \sqrt{\Sigma E_{\mathrm{T}}-350 \mathrm{GeV}}\right)^{2}\right]^{1 / 2} .
$$

As expected, the resolution is comparable to that observed in soft collisions (Fig. 4).

\section{Corrections to $\mathrm{E}_{\mathrm{T}}^{\mathrm{miss}}$}

Earlier studies [29] show that using calibrated jet energy scale can restore the average missing transverse energy scale in processes containing genuine $E_{\mathrm{T}}^{\text {miss }}$, but similar methods do not significantly reduce the fake reconstructed $E_{\mathrm{T}}^{\mathrm{miss}}$ in QCD events, which mainly comes from the stochastic effect of the calorimeter jet energy response.

Samples of $\mathrm{t} \overline{\mathrm{t}}$ and $\mathrm{W}+\mathrm{jet}$ with leptonic decays were used to investigate the reconstruction of events which contain a true $E_{\mathrm{T}}^{\text {miss }}$. Events having isolated muons or electrons with transverse momentum $\left(p_{\mathrm{T}}>20 \mathrm{GeV}\right)$ and pseudorapidity ( $|\eta|<3.0$ for electrons and $|\eta|<2.4$ for muons) were selected after full detector reconstruction. Jets are reconstructed using the iterative cone algorithm with a cone size $R=0.5$, seed threshold $E_{\mathrm{T}}=1.0 \mathrm{GeV}$, minimum jet threshold $E_{\mathrm{T}}=15 \mathrm{GeV}$, and calorimeter tower threshold $E_{\mathrm{T}}=0.5 \mathrm{GeV}$. Muons were reconstructed using the level 3 muon code from ORCA [15],[22]. Figure 16 shows the resulting jet $p_{\mathrm{T}}$ spectrum from QCD (solid line), $\mathrm{W}+$ jet with $\mathrm{W} p_{T}$ between 40 and $300 \mathrm{GeV}$ (dashed line). and $\mathrm{t} \overline{\mathrm{t}}$ (dotted line). Figure 17 shows the

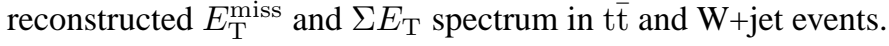

Corrections were made to the jet energy scale using the technique described in Ref. [28]. The main purpose of the correction, which restores the average reconstructed jet $E_{\mathrm{T}}$ in a cone to the average generated $E_{\mathrm{T}}$ in the cone, is to account for the nolinearity of the calorimetry. The size of the correction, which depends on $\eta$, is $10-20 \%$ at jet $p_{\mathrm{T}}=350 \mathrm{GeV} / c$. In basing the jet corrections on QCD events, which is done in order to suppress fake $E_{\mathrm{T}}^{\text {miss }}$ from badly reconstructed jets, we introduce a $p_{\mathrm{T}}$-dependent bias into the $\mathrm{t} \overline{\mathrm{t}}$ and $\mathrm{W}+\mathrm{jet}$ samples due to their different parton composition. The correction factors are calculated as a function of jet $p_{\mathrm{T}}$ and $\eta$ with QCD events Figure 18 shows the missing transverse energy resolution $v s$. reconstructed $E_{\mathrm{T}}^{\text {miss }}$ before (open circles) and after (closed circles) jet corrections for (a) $t \bar{t}$ events and and (b) $W+j e t$ events, Figure 19 shows the error in the reconstructed missing transverse energy scale $v s$. reconstructed $E_{\mathrm{T}}^{\mathrm{miss}}$ before (open circles) and after (closed circles) jet corrections for (a) t $\bar{t}$ events and (b) $\mathrm{W}+$ jet events. 
(a)

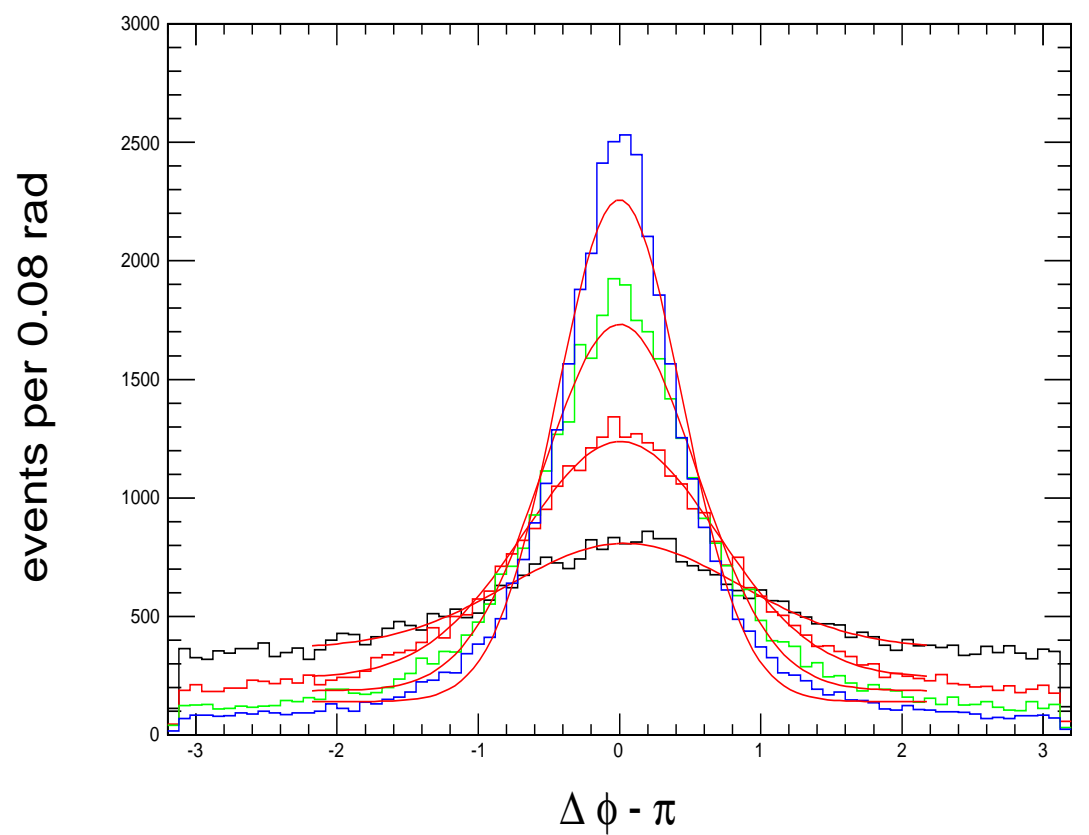

(b)

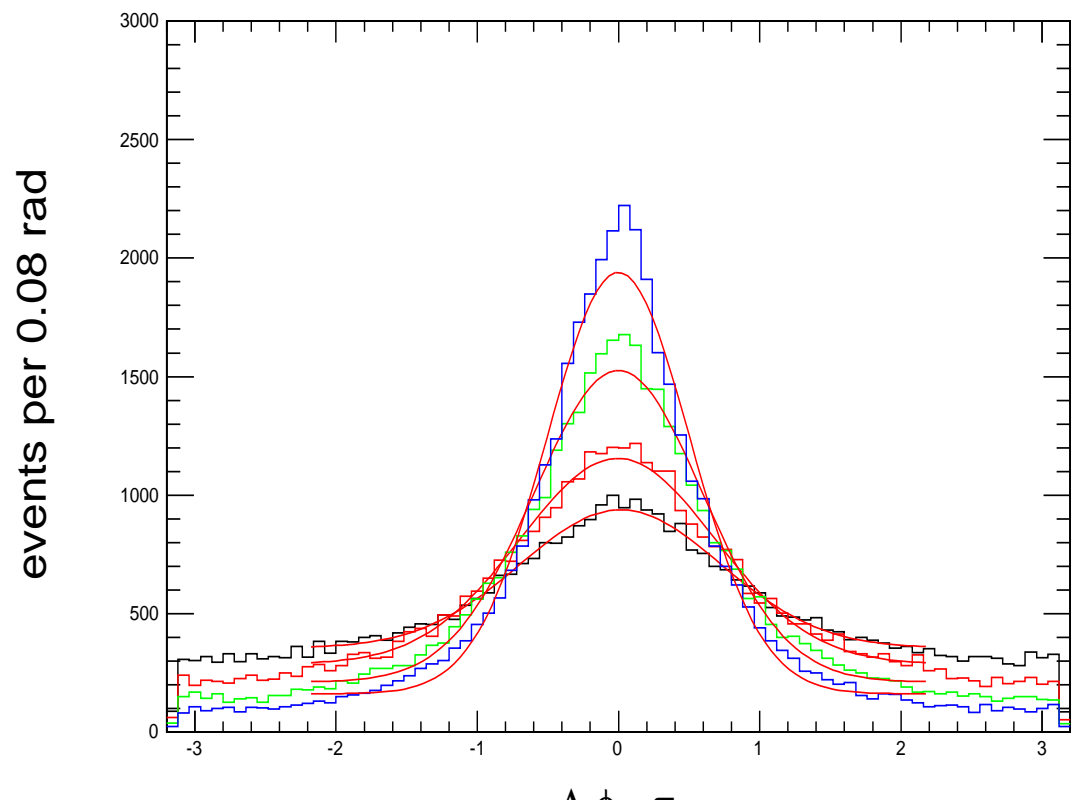

$\Delta \phi-\pi$

Figure 14: The $\varphi$ correlation between the missing transverse energy vectors reconstructed in the jet and unclustered regions for QCD samples with (a) $\hat{p}_{\mathrm{T}}$ of $30-50$ and (b) $50-80 \mathrm{GeV}$ for four cone radii $R=0.2,0.4,0.6$ and 0.8 , from low to high peak height, respectively. The distributions show that the vectors $\mathbf{E}_{\mathrm{Tj}}^{\mathrm{miss}}$ and $\mathbf{E}_{\mathrm{Tu}}^{\mathrm{miss}}$ are back-to-back. 


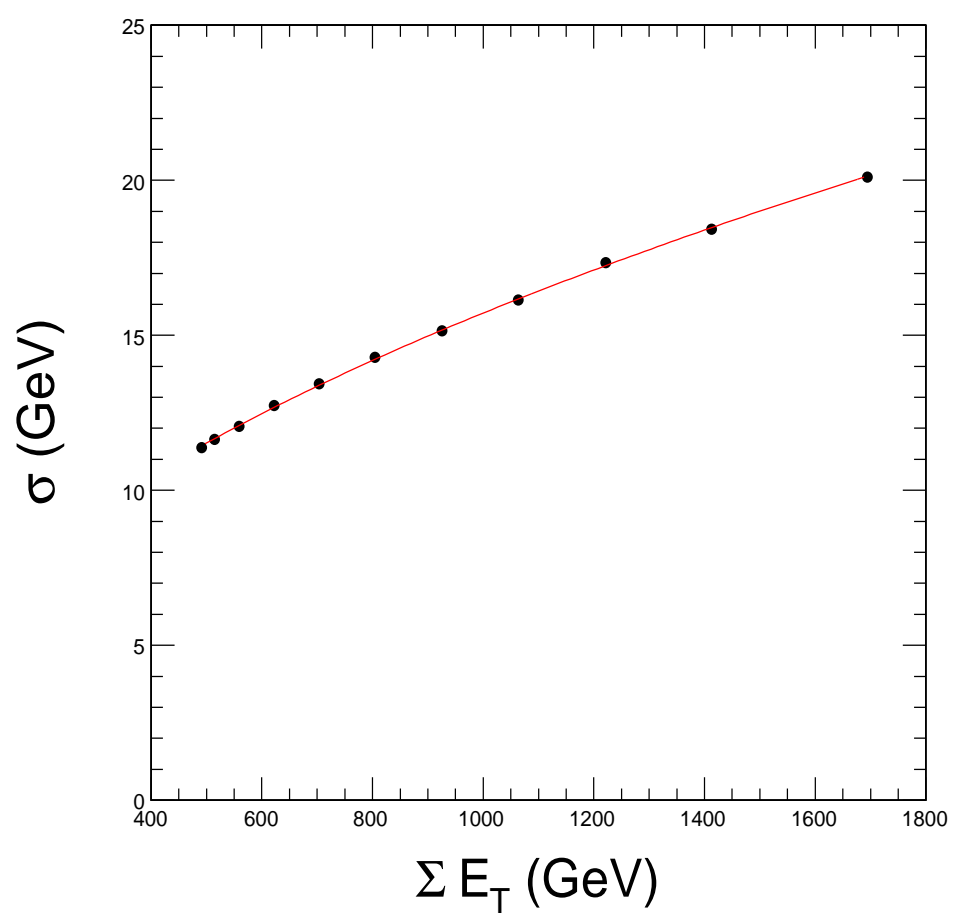

Figure 15: Missing transverse energy resolution in a direction orthogonal to the jet axis $v s . \Sigma E_{\mathrm{T}}$ of the entire event. The resolution is comparable to that observed in soft collisions (Fig 4). A fit (curve) gives $\sigma=\left[(9.9 \mathrm{GeV})^{2}+\right.$ $\left.\left(0.48 \mathrm{GeV}^{1 / 2} \sqrt{\Sigma E_{\mathrm{T}}-350 \mathrm{GeV}}\right)^{2}\right]^{1 / 2}$

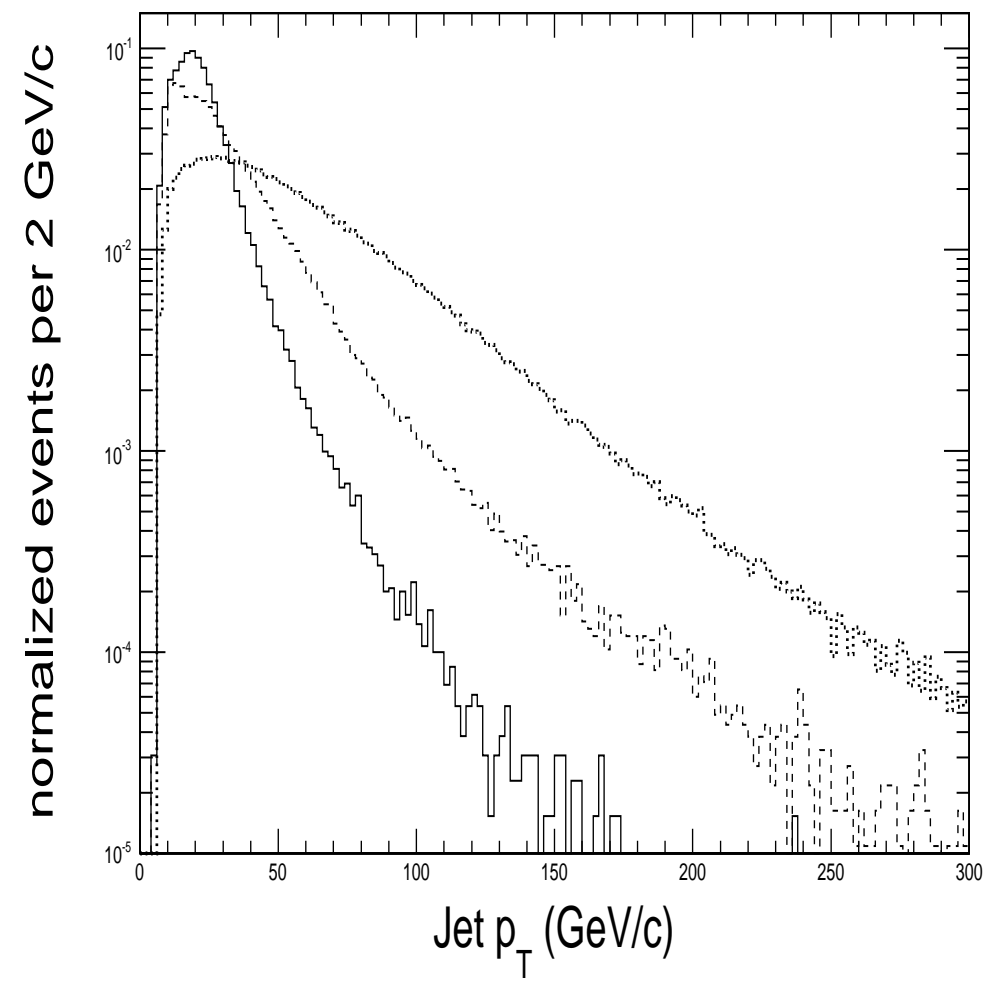

Figure 16: Jet $p_{\mathrm{T}}$ spectrum for events having an isolated lepton with $p_{\mathrm{T}}>20 \mathrm{GeV}$ from QCD (solid line), W+jet with $\mathrm{W} p_{\mathrm{T}}$ between 40 and $300 \mathrm{GeV}$ (dashed line). and tt (dotted line). 
(a)

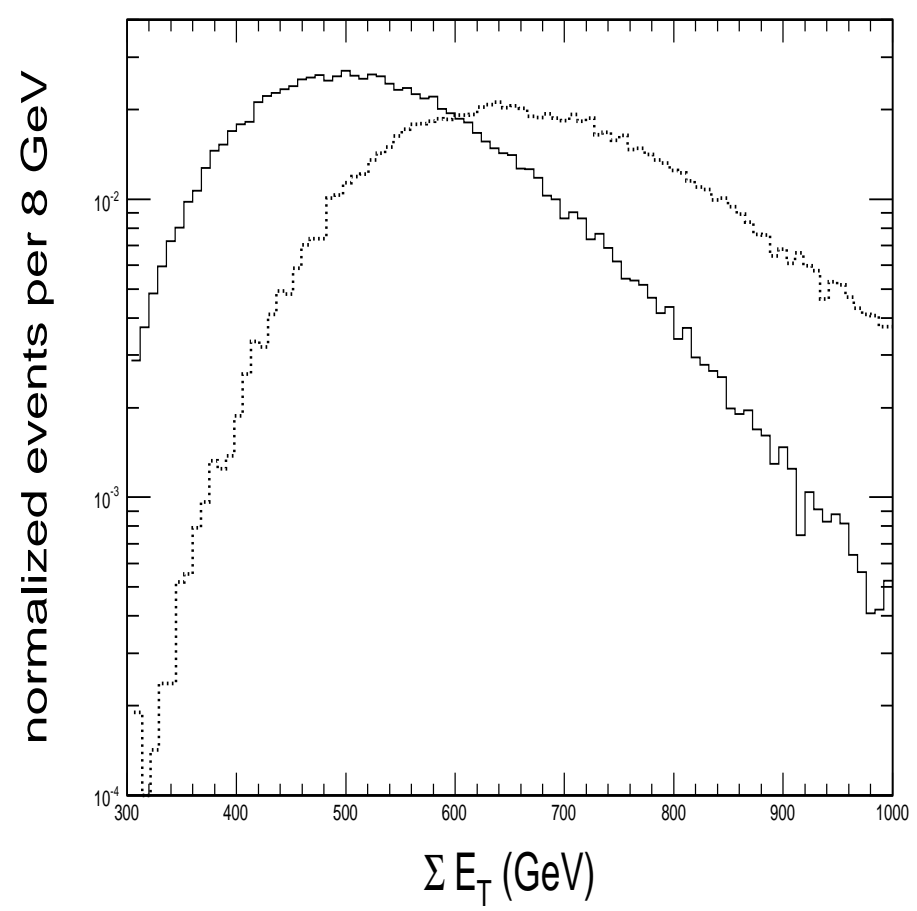

(b)

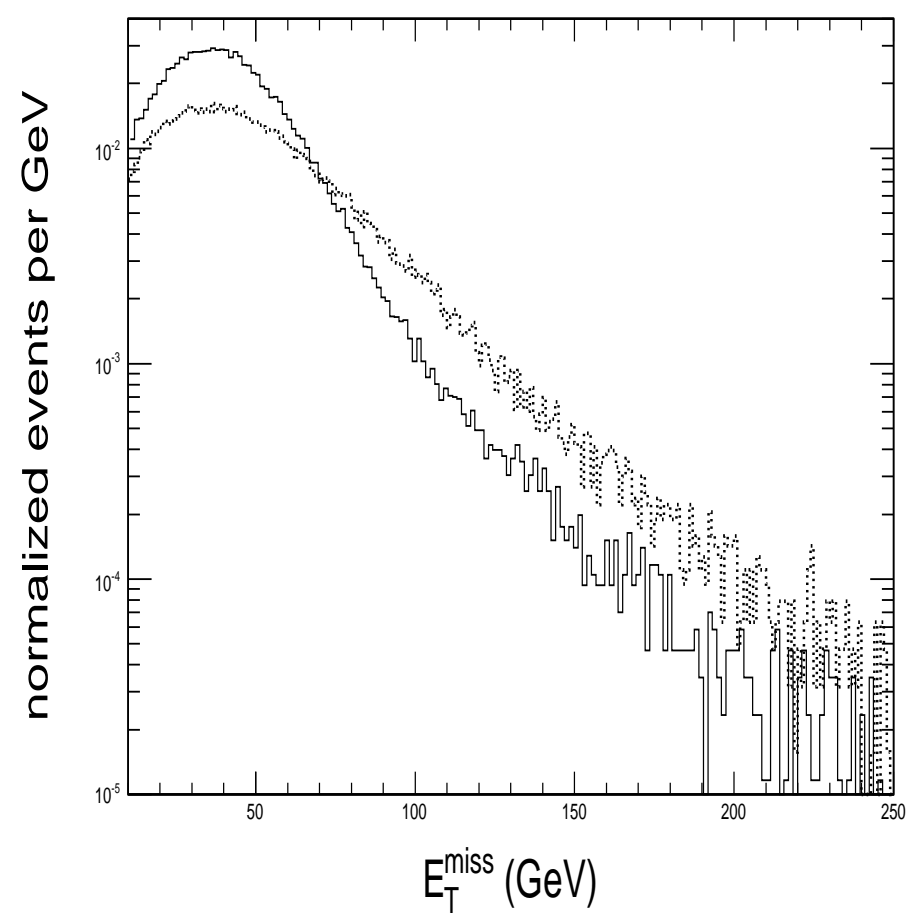

Figure 17: Reconstructed (a) $\Sigma E_{\mathrm{T}}$ and (b) $E_{\mathrm{T}}^{\mathrm{miss}}$ in $\mathrm{t} \overline{\mathrm{t}}$ events (dashed line) and $\mathrm{W}+\mathrm{jet}$ events with $\mathrm{W} p_{\mathrm{T}}$ between 40 and $300 \mathrm{GeV}$ (solid line). 


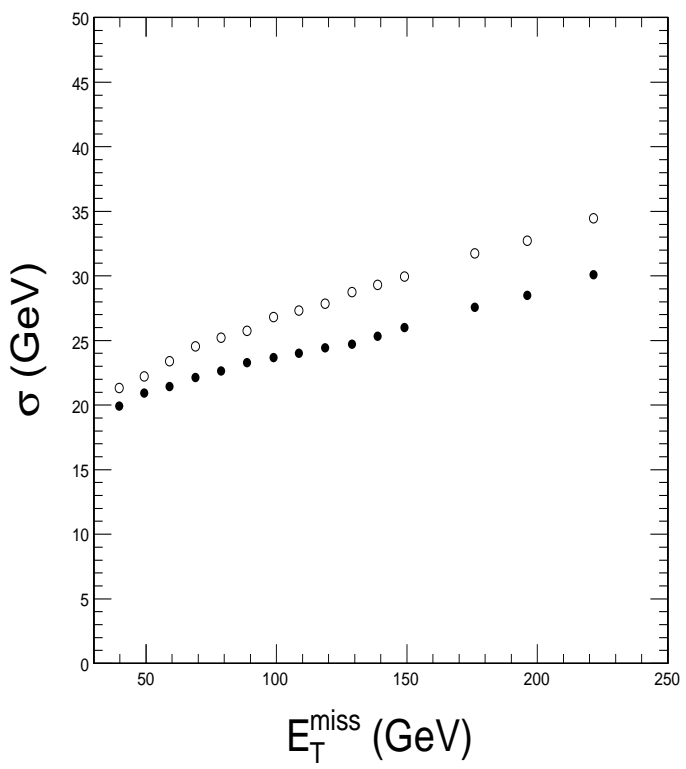

(a)

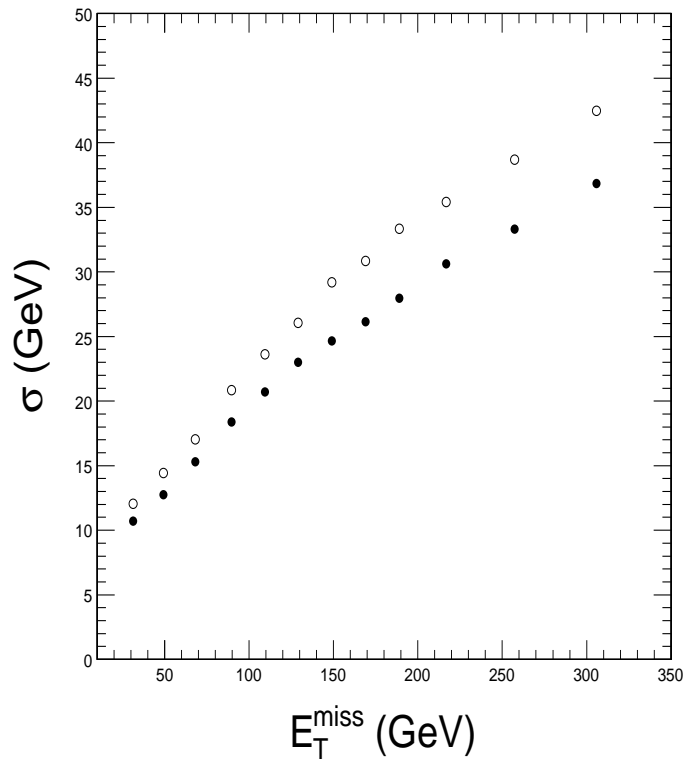

(b)

Figure 18: Missing transverse energy resolution before (open circles) and after (closed circles) jet corrections vs.

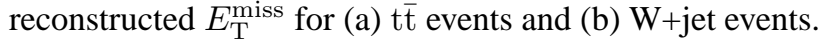

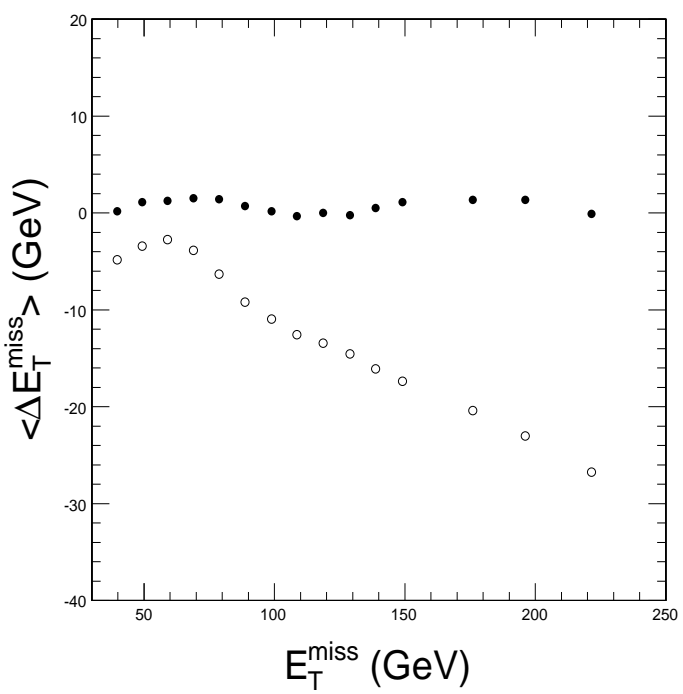

(a)

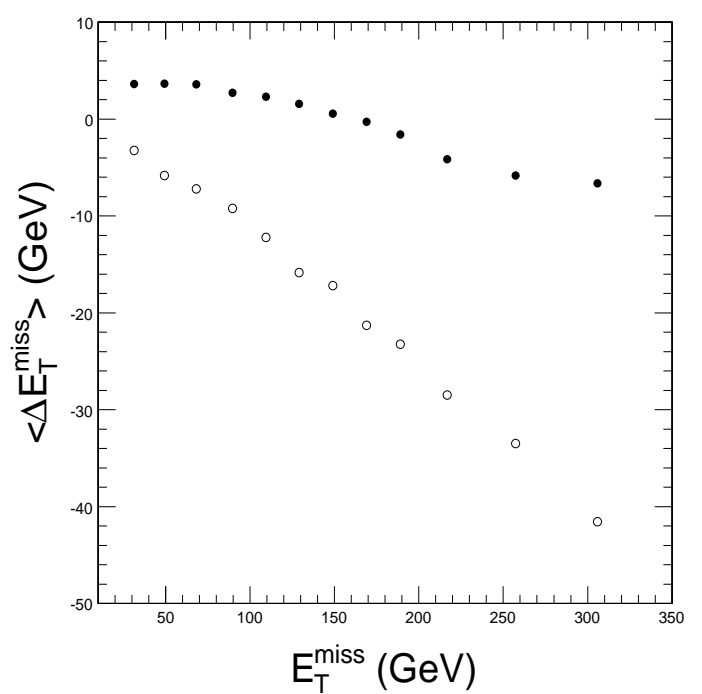

(b)

Figure 19: Error in the reconstructed missing transverse energy scale $v s$. reconstructed $E_{\mathrm{T}}^{\mathrm{miss}}$ before (open circles) and after (closed circles) jet corrections for (a) tét events and (b) W+jet events. 


\section{Angular Resolution}

To establish a signature for missing transverse energy in an event, it is likely necessary to show that the vector $\mathbf{E}_{\mathrm{T}}^{\text {miss }}$ does not have the same $\varphi$ as an energetic jet whose energy fluctuation in the calorimeter may well have been the cause of the reconstructed $E_{\mathrm{T}}^{\text {miss }}$. Figure 20(a) shows the $\mathbf{E}_{\mathrm{T}}^{\text {miss }}$ angular resolution as a function of reconstructed $E_{\mathrm{T}}^{\text {miss }}$ in $t \overline{\mathrm{t}}$ events with leptonic decays having $p_{\mathrm{T}}^{\ell}>20 \mathrm{GeV}$ before (open circles) and after (closed circles) Monte Carlo corrections to the jet energies. The resolution is calculated by fitting the distribution $\varphi_{\mathrm{r}}-\varphi_{\mathrm{g}}$, where $\varphi_{\mathrm{r}}\left(\varphi_{\mathrm{g}}\right)$ is the azimuthal angle of the reconstructed (generated) missing transverse energy vector. The angular resolution for measured $E_{\mathrm{T}}^{\text {miss }}=45 \mathrm{GeV}$ is comparable to the jet cone size $\left(0.5\right.$ radian) but improves at $E_{\mathrm{T}}^{\text {miss }}=100 \mathrm{GeV}$ to the jet core size ( 0.2 radian) [13]. At larger values of $E_{\mathrm{T}}^{\text {miss }}$, the resolution approaches that of the hadronic tower size ( 0.1 radian). The Monte Carlo corrections of the missing transverse energy vector make only a small improvement, approximately 0.02 radians for reconstructed $E_{\mathrm{T}}^{\mathrm{miss}}=100 \mathrm{GeV}$, to the angular resolution.

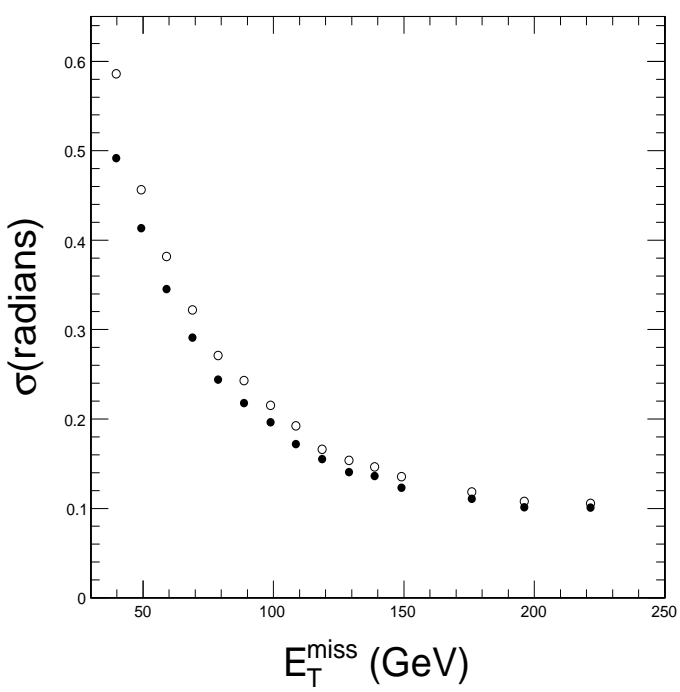

(a)

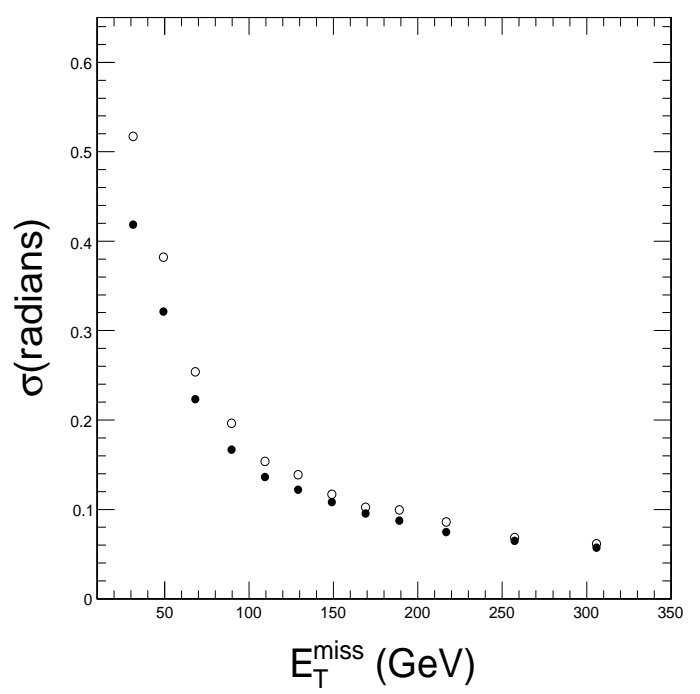

(b)

Figure 20: Phi resolution of the vector $\mathbf{E}_{\mathrm{T}}^{\text {miss }}$ as a function of reconstructed $E_{\mathrm{T}}^{\text {miss }}$ in events with leptonic decays having $p_{\mathrm{T}}^{\ell}>20 \mathrm{GeV}$ for (a) t $\overline{\mathrm{t}}$ and (b) $\mathrm{W}+$ jet events before (open circles) and after (closed circles) Monte Carlo corrections to the jet energies.

The missing transverse energy angular resolution has also been studied for $\mathrm{W}+\mathrm{jet}$ events with leptonic decays having $p_{\mathrm{T}}^{\ell}>20 \mathrm{GeV}$, which have a different topology than $\mathrm{t} \overline{\mathrm{t}}$ events. In these events, the $\mathrm{W} p_{\mathrm{T}}$ is approximately twice the average $p_{\mathrm{T}}$ of an energetic neutrino which causes a non-zero reconstructed missing transverse energy. In this case, the missing transverse energy tends to be opposite in $\varphi$ to an energetic jet whose mismeasurement contributes directly to the reconstructed $E_{\mathrm{T}}^{\text {miss }}$. Figure 20(b) shows the missing transverse energy $\varphi$ resolution as a

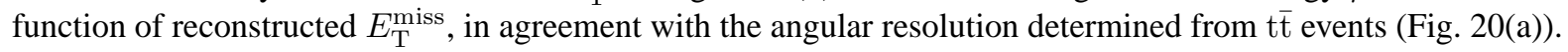

\section{Summary}

The missing transverse energy performance of the CMS detector has been evaluated using fully simulated minimum bias, QCD dijet, $t \bar{t}$, and $\mathrm{W}+$ jet events with high statistics. For minimum bias events without pileup, a resolution of $6.1 \mathrm{GeV}$ is computed, corresponding to a stochastic contribution of $0.63 \sqrt{\Sigma E_{\mathrm{T}}} \mathrm{GeV}^{1 / 2}$, where $\Sigma E_{\mathrm{T}}$ is the summed transverse energy in all calorimeter towers. This result is in agreement with expectations from measurements at UA1 and CDF, taking into account differences in calorimeter resolution. When the contribution of pileup is included, the resolution degrades according to the overall deposited $\Sigma E_{\mathrm{T}}$ with the same stochastic coefficient. For QCD events, we expect $\sigma=\left[(3.8 \mathrm{GeV})^{2}+\left(0.97 \mathrm{GeV}^{1 / 2} \sqrt{\Sigma E_{\mathrm{T}}}\right)^{2}+\left(0.012 \Sigma E_{\mathrm{T}}\right)^{2}\right]^{1 / 2}$ resulting in a resolution of $45 \mathrm{GeV}$ for jet events with reconstructed transverse momentum of $800 \mathrm{GeV} / c$, again in accord with measurements made at UA1 and CDF. A sample of $t \bar{t}$ events with lepton decays leading to true missing transverse energy was used to determine the azimuthal angle resolution to be 0.1 radians $(0.2$ radians) for a reconstructed missing transverse energy of $200 \mathrm{GeV}(100 \mathrm{GeV})$. 


\section{References}

[1] “LHC Design Report,” Vols. I and II, http://lhc.web.cern.ch/lhc/.

[2] A. Astbury et al., "A 4 $4 \pi$ Solid-Angle Detector for the SPS Used as a Proton-Antiproton Collider at a Centreof-Mass Energy of $540 \mathrm{GeV}$," CERN/SPSC 78-06 (1978).

[3] “CMS, The Hadron Calorimeter Technical Design Report,” CERN/LHCC 97-31 CMS TDR 2 (June 1997).

[4] The Compact Muon Solenoid, Letter of Intent, CERN/LHCC 92-3, LHCC/I 1, 1 October 1992.

[5] G. Arnison et al., "Experimental Observation of Isolated Large Transverse Energy Electrons With Associated Missing Energy at $\sqrt{s}=540 \mathrm{GeV}$," Phys. Lett. 122B (1983) 103.

[6] G. Arnison et al., "Events With Large Missing Transverse Energy at the CERN Collider: I. W $\rightarrow \tau \nu$ Decay and Test of $\tau-\mu-\mathrm{e}$ Universality at $Q^{2}=m_{\mathrm{W}^{2}}$," Phys. Lett. 185B (1987) 233; G. Arnison et al., "Events With Large Missing Transverse Energy at the CERN Collider: II. Search for the Decays of $\mathrm{W}^{ \pm}$Into Heavy Leptons and of $Z^{0}$ Into Non-Interacting Particles", Phys. Lett. 185B (1987) 241; G. Arnison et al., "Events With Large Missing Transverse Energy at the CERN Collider: III. Mass Limits on Supersymmetric Particles," Phys. Lett. 198B (1987) 261.

[7] S. Abachi et al., "Search for Squarks and Gluinos in pp Collisions at $\sqrt{s}=1.8-\mathrm{TeV}$," Phys. Rev. Lett. 75 (1995) 618.

[8] F. Abe et al., "Search for Gluinos and Squarks at the Fermilab Tevatron Collider," Phys. Rev. D56 (1997) 1357.

[9] M. Spiropulu, "A Blind Search for Supersymmetry in p $\bar{p}$ Collisions at $\sqrt{s}=1.8-\mathrm{TeV}$ Using the Missing Energy Plus Multijet Channel," FERMILAB-THESIS-2000-16.

[10] "CMS, The Electromagnetic Calorimeter Technical Design Report," CERN/LHCC 97-33, CMS TDR 4 (1997), Addendum CERN/LHCC 2002-27 (2002).

[11] G. Alverson, G. Eulisse, S. Muzaffar, I. Osborne, L. Taylor, and L. A. Tuura, "IGUANA: A High-Performance 2D and 3D Visualisation System," Nucl. Instrum. and Meth. A534 (2004) 143.

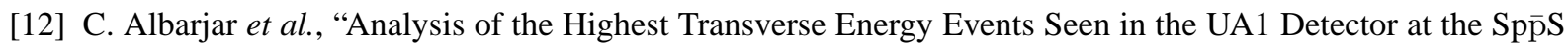
Collider,” Z. Phys. C36 (1987) 33.

[13] G. Arnison et al., "Hadronic Jet Production at the Cern Proton-Antiproton Collider," Phys. Lett. 132B (1983) 214.

[14] F. Abe et al., "Measurement of the W-boson Mass," Phys. Rev. Lett. 65 (1990) 2243. submitted to Nucl. Inst. and Meth.

[15] CMS, The TriDAS Project, Technical Design Report, Volume 2: Data Acquisition and High-Level Trigger, CERN/LHCC 2002-26, CMS TDR 6.2 (2002).

[16] CMS Collaboration, “The Computing Project Technical Design Report,” CERN/LHCC 2005-23 (2005), CMS TDR 7.

[17] H. Pi, "Reconstruction of Missing Transverse Energy and Prospect of Searching for Higgs Boson Produced via Vector Boson Fusion in Compact Muon Solenoid Expemiment," Ph.D. thesis, U. Florida (2006).

[18] T. Sjstrand, P. Edn, C. Friberg, L. Lnnblad, G. Miu, S. Mrenna and E. Norrbin, "High-Energy-Physics Event Generation with PYTHIA 6.1," Computer Phys. Commun. 135 (2001) 238 (LU TP 00-30, hep-ph/0010017).

[19] V. Karimäki, “CMKIN, CMS Physics Generators Interface," http://cmsdoc.cern.ch/cmsoo/projects/CMKIN/.

[20] GEANT4 Collaboration, S. Agostinelli et al., "GEANT4: A Simulation Toolkit,” Nucl. Instr. and Meth. A506 (2003) 250.

[21] M. Stavrianakou, "OSCAR: Object-oriented Simulation for CMS Analysis and Reconstruction," http://cmsdoc.cern.ch/OSCAR/.

[22] S. Wynhoff, “ORCA: CMS Object-Oriented Reconstruction,” http://cmsdoc.cern.ch/orca/. 
[23] E. James, Y. Maravin, M. Mulders, and N. Neumeister, "Muon Identification in CMS," CMS Note 2006/10 (2006).

[24] H. Pi, “CMS JetMET Datasets in DC04 and Post-DC04," http://cms00.phys.ufl.edu/cms/DC04/PCP/.

[25] O. Kodolova et al., "Jet Energy Correction with Charged Particle Tracks in CMS," ”Eur. Phys. J. C40S2 (2005) 33.

[26] ATLAS Collaboration, "ATLAS Detector and Physics Performance Technical Design Report," CERN/LHCC 99-14 (1999) ATLAS TDR 14.

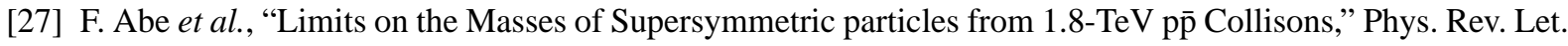
62 (1989) 1825

[28] A. Heister et al., "Measurement of Jets With the CMS Detector at the LHC," CMS Note 2006/036.

[29] A. Nikitenko, S. Kunori, and R. Kinnunen, "Missing Transverse Energy Measurement with Jet Energy Corrections," CMS NOTE 2001/040 (2001). 